TRANSACTIONS OF THE

AMERICAN MATHEMATICAL SOCIETY

Volume 352 , Number 6 , Pages $2825-2855$

S 0002-9947(00)02472-7

Article electronically published on February 28, 2000

\title{
THE TRUNCATED COMPLEX $K$-MOMENT PROBLEM
}

\author{
RAÚL E. CURTO AND LAWRENCE A. FIALKOW \\ Dedicated to Professor Aaron D. Fialkow \\ on the occasion of his eighty-seventh birthday
}

\begin{abstract}
Let $\gamma \equiv \gamma^{(2 n)}$ denote a sequence of complex numbers $\gamma_{00}, \gamma_{01}, \gamma_{10}, \ldots, \gamma_{0,2 n}, \ldots, \gamma_{2 n, 0}\left(\gamma_{00}>0, \gamma_{i j}=\bar{\gamma}_{j i}\right)$, and let $K$ denote a closed subset of the complex plane $\mathbb{C}$. The Truncated Complex $K$-Moment Problem for $\gamma$ entails determining whether there exists a positive Borel measure $\mu$ on $\mathbb{C}$ such that $\gamma_{i j}=\int \bar{z}^{i} z^{j} d \mu(0 \leq i+j \leq 2 n)$ and $\operatorname{supp} \mu \subseteq K$. For $K \equiv K_{\mathcal{P}}$ a semi-algebraic set determined by a collection of complex polynomials $\mathcal{P}=\left\{p_{i}(z, \bar{z})\right\}_{i=1}^{m}$, we characterize the existence of a finitely atomic representing measure with the fewest possible atoms in terms of positivity and extension properties of the moment matrix $M(n)(\gamma)$ and the localizing matrices $M_{p_{i}}$. We prove that there exists a rank $M(n)$-atomic representing measure for $\gamma^{(2 n)}$ supported in $K_{\mathcal{P}}$ if and only if $M(n) \geq 0$ and there is some rankpreserving extension $M(n+1)$ for which $M_{p_{i}}\left(n+k_{i}\right) \geq 0$, where $\operatorname{deg} p_{i}=2 k_{i}$ or $2 k_{i}-1(1 \leq i \leq m)$.
\end{abstract}

\section{INTRODUCTION}

Let $\gamma \equiv \gamma^{(2 n)}$ denote a sequence of complex numbers $\gamma_{00}, \gamma_{01}, \gamma_{10}, \ldots, \gamma_{0,2 n}$, $\ldots, \gamma_{2 n, 0}\left(\gamma_{00}>0, \gamma_{i j}=\bar{\gamma}_{j i}\right)$, and let $K$ denote a closed subset of the complex plane $\mathbb{C}$. The Truncated Complex $K$-Moment Problem for $\gamma$ entails determining whether there exists a positive Borel measure $\mu$ on $\mathbb{C}$ such that

$$
\gamma_{i j}=\int \bar{z}^{i} z^{j} d \mu \quad(0 \leq i+j \leq 2 n)
$$

and

$$
\operatorname{supp} \mu \subseteq K
$$

a measure $\mu$ satisfying (1.1) is a representing measure for $\gamma$.

In the sequel, we characterize the existence of a representing measure satisfying (1.2) in the case when $K$ is a semi-algebraic set determined by a finite collection of

Received by the editors May 14, 1998.

2000 Mathematics Subject Classification. Primary 47A57, 44A60, 30E05; Secondary 15A57, 15-04, 47N40, 47A20.

Key words and phrases. Truncated complex moment problem, moment matrix extension, flat extensions of positive matrices, semi-algebraic sets, localizing matrix.

Research partially supported by NSF grants. The second-named author was also partially supported by the State University of New York at New Paltz Research and Creative Projects Award Program. 
complex polynomials $\mathcal{P}=\left\{p_{i}(z, \bar{z})\right\}_{i=1}^{m}$, i.e.,

$$
K=K_{\mathcal{P}}:=\left\{z \in \mathbb{C}: p_{i}(z, \bar{z}) \geq 0,1 \leq i \leq m\right\} .
$$

Our existence criterion is expressed in terms of positivity and extension properties of the moment matrix $M(n)(\gamma)$ associated to $\gamma$ and of the localizing matrix $M_{p_{i}}$ corresponding to each $p_{i}$ (see below).

This research is motivated by the extensive literature concerning the Full Multivariable $K$-Moment Problem [Akh], Fug], [Hav], [Sch], $[\mathrm{ShTa}$, including the Full Complex $K$-Moment Problem in which moments of all orders are prescribed, i.e., $\gamma=\left(\gamma_{i j}\right)_{i, j \geq 0}$. The Riesz-Haviland criterion [Rie, $\left.\S 5\right]$, [Hav] provides an "abstract" solution to the Full Multivariable $K$-Moment Problem. In the case of the Full Complex $K$-Moment Problem this criterion may be expressed as follows:

$$
p(z, \bar{z}) \equiv \sum a_{r s} \bar{z}^{r} z^{s} \in \mathbb{C}[z, \bar{z}],\left.p\right|_{K} \geq 0 \Longrightarrow \sum a_{r s} \gamma_{r s} \geq 0 .
$$

For general closed sets $K \subset \mathbb{C}$, the cone of nonnegative polynomials cannot easily be characterized, so the Riesz-Haviland criterion is intractable. In particular, for $K=\mathbb{C}$ there is no concrete description of the nonnegative polynomials, and the Full Complex Moment Problem (equivalent to the Full Two-Dimensional Real Moment Problem) remains unsolved. By contrast, A. Atzmon [Atz] found a "concrete" solution to the Full Complex Moment Problem for $K=\overline{\mathbb{D}}$, the closed unit disk; an alternate solution to the disk problem was formulated by M. Putinar Put]. Subsequently, K. Schmüdgen [Sch] presented a concrete solution to the Full Multivariable $K$-Moment Problem in the case when $K$ is a general compact semi-algebraic set ( $c f$. the remarks following Theorem 1.8 below).

A result of J. Stochel [Sto] shows that the Truncated Multivariable $K$-Moment Problem is more general than the Full Multivariable $K$-Moment Problem; nevertheless, it appears that prior to the present work there has been no systematic study of truncated multivariable $K$-moment problems. The criterion that we develop for general semi-algebraic sets is not as directly applicable as Schmüdgen's criterion for the compact semi-algebraic case, but whenever our moment matrix method can be applied, it yields an explicit formula for a finitely atomic representing measure supported in $K$. In a companion paper [CuFi7], we use moment matrix techniques independent of the present work to explicitly solve the Quadratic Moment Problem $(n=1)$ for the unit disk and the unit circle.

We devote the remainder of this section to stating the main results and illustrating them with examples. Section 2 contains a brief survey of some necessary background results on moment matrices. In Section ?? we introduce localizing matrices, the new tool that we employ to locate the support of a representing measure relative to a prescribed semi-algebraic set; Section ?? contains the proof of Theorem 1.2. which describes localizing matrices in terms of compressions of moment matrices. Section 4 contains the proofs of the existence theorems for finitely atomic representing measures supported in semi-algebraic sets. All of these results for truncated $K$-moment problems in one complex variable can be generalized to any number of real or complex variables ( $c f$. [CuFi4, Chapter 7]); for the case of one real variable, in Section 5 we present new accounts of the truncated $K$-moment problems of Hamburger, Stieltjes, and Hausdorff for $K=\mathbb{R}, K=[0,+\infty)$, and $K=[a, b]$, respectively.

Let $\mathcal{P}_{n}$ denote the complex polynomials $q(z, \bar{z})=\sum a_{i j} \bar{z}^{i} z^{j}$ of total degree at most $n$, and for $q \in \mathcal{P}_{n}$, let $\hat{q}=\left(a_{i j}\right)$ denote the coefficient vector of $q$ with respect 
to the basis $\left\{\bar{z}^{i} z^{j}\right\}_{0 \leq i+j \leq n}$ of $\mathcal{P}_{n}$ (ordered lexicographically: $1, z, \bar{z}, z^{2}, z \bar{z}, \bar{z}^{2}, \ldots$, $\left.z^{n}, \ldots, \bar{z}^{n}\right)$. For $p \in \mathcal{P}_{2 n}, p(z, \bar{z}) \equiv \sum b_{i j} \bar{z}^{i} z^{j}$, let $\Lambda(p):=\sum b_{i j} \gamma_{i j}$. The moment matrix $M(n) \equiv M(n)(\gamma)$ is the unique matrix (of size $\frac{(n+1)(n+2)}{2}$ ) such that

$$
\langle M(n) \hat{f}, \hat{g}\rangle=\Lambda(f \bar{g}) \quad\left(f, g \in \mathcal{P}_{n}\right) .
$$

If we label the rows and columns of $M(n)$ lexicographically as $1, Z, \bar{Z}, Z^{2}, Z \bar{Z}$, $\bar{Z}^{2}, \ldots, Z^{n}, \ldots, \bar{Z}^{n}$, it follows that the row $\bar{Z}^{k} Z^{l}$, column $\bar{Z}^{i} Z^{j}$ entry of $M(n)$ is equal to

$$
\left\langle M(n) \widehat{\bar{z}^{i} z^{j}}, \widehat{\bar{z}^{k} z^{l}}\right\rangle=\Lambda\left(\bar{z}^{i+l} z^{j+k}\right)=\gamma_{i+l, j+k} .
$$

For example, with $n=1$, the Quadratic Moment Problem for $\gamma^{(2)}: \gamma_{00}, \gamma_{01}, \gamma_{10}$, $\gamma_{02}, \gamma_{11}, \gamma_{20}$ corresponds to

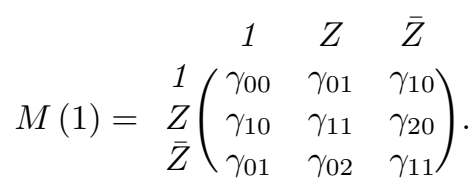

$\gamma$ admits a representing measure $\mu$, then for $f \in \mathcal{P}_{n},\langle M(n) \hat{f}, \hat{f}\rangle=\Lambda\left(|f|^{2}\right)=$ $\int|f|^{2} d \mu \geq 0$, whence $M(n) \geq 0$.

Now let $p \in \mathcal{P}_{2 n}, p \not \equiv 0$, and define $k$ by $\operatorname{deg} p=2 k$ or $\operatorname{deg} p=2 k-1$. There exists a unique localizing matrix $M_{p}(n) \equiv M_{p}(n)(\gamma)$ (of size $\frac{(n-k+1)(n-k+2)}{2}$ ) such that

$$
\left\langle M_{p}(n) \hat{f}, \hat{g}\right\rangle=\Lambda(p f \bar{g}) \quad\left(f, g \in \mathcal{P}_{n-k}\right) .
$$

Thus, if a representing measure $\mu$ for $\gamma$ is supported in $K_{p} \equiv\{z \in \mathbb{C}: p(z, \bar{z}) \geq 0\}$, then for $f \in \mathcal{P}_{n-k}$,

$$
\left\langle M_{p}(n) \hat{f}, \hat{f}\right\rangle=\Lambda\left(p|f|^{2}\right)=\int p|f|^{2} d \mu \geq 0,
$$

whence $M_{p}(n) \geq 0$.

If $\gamma$ admits a representing measure $\mu$, then $\operatorname{card} \operatorname{supp} \mu \geq \operatorname{rank} M(n)$ CuFi4. Corollary 3.7]. The main result of [CuFi4] shows that $\gamma$ admits a rank $M(n)$ atomic (minimal) representing measure if and only if $M(n) \geq 0$ and $M(n)$ admits an extension to a (necessarily positive) moment matrix $M(n+1)$ satisfying $\operatorname{rank} M(n+1)=\operatorname{rank} M(n)$; such an extension is called a flat extension. A variety of concrete conditions for the existence of flat extensions are presented in [CuFi5], [CuFi6], though complete necessary and sufficient conditions are not known.

The Flat Extension Theorem (Theorem 2.1 below, $c f$. [CuFi4, [Fi3]) shows that if $M(n) \geq 0$ admits a flat extension $M(n+1)$, then $M(n+1)$ admits unique successive flat (positive) moment matrix extensions $M(n+2), M(n+3), \ldots$ Further, let $r:=\operatorname{rank} M(n)$, so that in $\mathcal{C}_{M(r)}$ (the column space of $M(r)$ ) there is a dependence relation of the form $Z^{r}=c_{0} 1+c_{1} Z+\cdots+c_{r-1} Z^{r-1}$. The polynomial $z^{r}-\left(c_{0}+\cdots+c_{r-1} z^{r-1}\right)$ has $r$ distinct roots, $z_{0}, \ldots, z_{r-1}$, which provide the support for the unique representing measure for $\gamma^{(2 n+2)}$ corresponding to the flat extension $M(n+1)$. The densities of this measure, $\rho_{0}, \ldots, \rho_{r-1}$, are determined by the Vandermonde equation

$$
V\left(z_{0}, \ldots, z_{r-1}\right)\left(\rho_{0}, \ldots, \rho_{r-1}\right)^{t}=\left(\gamma_{00}, \ldots, \gamma_{0, r-1}\right)^{t} ;
$$

we denote the measure $\sum_{i=0}^{r-1} \rho_{i} \delta_{z_{i}}$ by $\nu[M(n+1)]$. 
Our main result, which follows, characterizes the existence of a $\operatorname{rank} M(n)$ atomic representing measure supported in a semi-algebraic set $K_{p}$, where $p$ is an arbitrary polynomial in $\mathbb{C}[z, \bar{z}]$. Assume that $M(n) \geq 0$ admits a flat extension $M(n+1)$ and let $k:=\left[\frac{\operatorname{deg} p+1}{2}\right]$. Since $\operatorname{deg} p \leq 2(n+k), M_{p}(n+k)$ is well-defined relative to the unique flat extension $M(n+k)$ of $M(n+1)$ if $k \geq 1$, or relative to $M(n)$ if $k=0$.

Theorem 1.1. Let $p \in \mathbb{C}[z, \bar{z}], p \not \equiv 0, \operatorname{deg} p=2 k$ or $\operatorname{deg} p=2 k-1$. There exists a rank $M(n)$-atomic (minimal) representing measure for $\gamma^{(2 n)}$ supported in $K_{p}$ if and only if $M(n) \geq 0$ and there is some flat extension $M(n+1)$ for which $M_{p}(n+k) \geq 0$. In this case, the measure $\nu[M(n+1)]$ is a rank $M(n)$-atomic representing measure supported in $K_{p}$, with precisely $\operatorname{rank} M(n)-\operatorname{rank} M_{p}(n+k)$ atoms in $\mathcal{Z}(p) \equiv\{z \in \mathbb{C}: p(z, \bar{z})=0\}$.

To apply Theorem 1.1, we need to be able to explicitly compute localizing matrices. Suppose $p \in \mathcal{P}_{2 n}, d:=\operatorname{deg} p$, and let $k:=\left[\frac{d+1}{2}\right]$, so that $0 \leq k \leq n$. Write $p(z, \bar{z}) \equiv \sum_{0 \leq r+s \leq d} a_{r s} \bar{z}^{r} z^{s}$. Then

$$
\begin{aligned}
& \text { for each } r, s \text {, there exist } i, j \geq 0,0 \leq i+j \leq k, \\
& 0 \leq(r+s)-(i+j) \leq k \text {, such that } \bar{z}^{r} z^{s}=\bar{z}^{i} z^{j} \cdot \bar{z}^{r-i} z^{s-j}
\end{aligned}
$$

this decomposition is not unique. Let $\sigma=\frac{(n-k+1)(n-k+2)}{2}$, and let $\left[\bar{Z}^{s-j} Z^{r-i}\right]^{M(n)}{ }_{\left[\bar{Z}^{i} Z^{j}\right]}$ denote the compression of $M(n)$ to the first $\sigma$ columns indexed by multiples of $\bar{Z}^{i} Z^{j}$ and to the first $\sigma$ rows indexed by multiples of $\bar{Z}^{s-j} Z^{r-i}$. The following result provides a computational formula which expresses $M_{p}(n)$ as a linear combination of compressions of $M(n)$.

Theorem 1.2. Let $p \in \mathcal{P}_{2 n}, p \not \equiv 0, p(z, \bar{z}) \equiv \sum_{0 \leq r+s \leq d} a_{r s} \bar{z}^{r} z^{s}$. Then

$$
M_{p}(n)=\sum_{0 \leq r+s \leq d} a_{r s}\left[\bar{Z}^{s-j} Z^{r-i}\right]{ }^{M(n)}\left[\bar{Z}^{i} Z^{j}\right] .
$$

Moment matrix structure implies that the expression for $M_{p}(n)$ in Theorem 1.2 is independent of the decomposition in (1.5).

In the sequel, we denote the compression of a moment matrix $M(n)$ to rows $\bar{Z}^{i_{1}} Z^{j_{1}}, \ldots, \bar{Z}^{i_{p}} Z^{j_{p}}$ and columns $\bar{Z}^{r_{1}} Z^{s_{1}}, \ldots, \bar{Z}^{r_{q}} Z^{s_{q}}$ by

$$
\left\{\bar{Z}^{i_{1}} Z^{j_{1}}, \ldots, \bar{Z}^{i_{p}} Z^{j_{p}}\right\}^{M(n)}\left\{\bar{Z}^{r_{1}} Z^{s_{1}}, \ldots, \bar{Z}^{r_{q}} Z^{s_{q}}\right\} \cdot
$$

For a square matrix $M$ and $p>0$, we let $[M]_{p}$ denote the compression of $M$ to the first $p$ rows and columns. We let $M_{\left\{p_{1}, \ldots, p_{k}\right\}}$ denote the compression of $M$ to rows and columns $p_{1}, \ldots, p_{k}$.

We illustrate Theorem 1.2 by computing $M_{p}(2)$ for $p(z, \bar{z})=1-z \bar{z}$.

Example 1.3. Let

$$
\left.M \equiv M(2)=\begin{array}{c}
1 \\
1 \\
Z \\
\bar{Z}
\end{array} \begin{array}{cccccc}
\gamma_{00} & \gamma_{01} & \gamma_{10} & \gamma_{02} & \gamma_{11} & \gamma_{20} \\
Z_{10} & \gamma_{11} & \gamma_{20} & \gamma_{12} & \gamma_{21} & \gamma_{30} \\
\gamma_{01} & \gamma_{02} & \gamma_{11} & \gamma_{03} & \gamma_{12} & \gamma_{21} \\
\gamma_{20} & \gamma_{21} & \gamma_{30} & \gamma_{22} & \gamma_{31} & \gamma_{40} \\
\bar{Z}_{11} & \gamma_{12} & \gamma_{21} & \gamma_{13} & \gamma_{22} & \gamma_{31} \\
\gamma_{02} & \gamma_{03} & \gamma_{12} & \gamma_{04} & \gamma_{13} & \gamma_{22}
\end{array}\right)
$$


and let $p(z, \bar{z}):=1-z \bar{z}$. We compute $M_{p}(2)$ using $n=2, k=1, \sigma=3,1=1 \cdot 1$, $z \bar{z}=\bar{z}^{0} z^{1} \cdot \bar{z}^{1} z^{0}(c f$. (1.5) $)$. Theorem [1.2 implies

$$
\begin{aligned}
M_{p}(2)= & \{1, Z, \bar{Z}\} M_{\{1, Z, \bar{Z}\}}-\left\{Z, Z^{2}, Z \bar{Z}\right\} M_{\left\{Z, Z^{2}, Z \bar{Z}\right\}} \\
= & \left(\begin{array}{lll}
\gamma_{00} & \gamma_{01} & \gamma_{10} \\
\gamma_{10} & \gamma_{11} & \gamma_{20} \\
\gamma_{01} & \gamma_{02} & \gamma_{11}
\end{array}\right)-\left(\begin{array}{lll}
\gamma_{11} & \gamma_{12} & \gamma_{21} \\
\gamma_{21} & \gamma_{22} & \gamma_{31} \\
\gamma_{12} & \gamma_{13} & \gamma_{22}
\end{array}\right) \\
= & \left(\begin{array}{lll}
\gamma_{00}-\gamma_{11} & \gamma_{01}-\gamma_{12} & \gamma_{10}-\gamma_{21} \\
\gamma_{10}-\gamma_{21} & \gamma_{11}-\gamma_{22} & \gamma_{20}-\gamma_{31} \\
\gamma_{01}-\gamma_{12} & \gamma_{02}-\gamma_{13} & \gamma_{11}-\gamma_{22}
\end{array}\right) .
\end{aligned}
$$

Note that the alternate decomposition $z \bar{z}=\bar{z}^{1} z^{0} \cdot \bar{z}^{0} z^{1}$ leads to the same result, as required.

We next illustrate Theorem 1.1 by adapting a result of [Fi3] which parameterizes the minimal quadrature rules of degree 2 for planar Lebesgue measure on the closed unit disk $\overline{\mathbb{D}}$.

Example 1.4. ([Fi3] $)$ The complex moments up to degree 2 of Lebesgue measure on the unit disk are $\gamma_{00}=\pi, \gamma_{11}=\frac{\pi}{2}, \gamma_{01}=\gamma_{10}=\gamma_{02}=\gamma_{20}=0$; in particular, $M(1)>0$. We seek to describe the representing measures for $\gamma^{(2)}$ that are supported in the closed disk and have minimal support among such measures. For each choice of "new moments" of degree 3 for such a measure, $\gamma_{12}=x$ and $\gamma_{03}=y$, let

$$
B=\left(\begin{array}{lll}
\gamma_{02} & \gamma_{11} & \gamma_{20} \\
\gamma_{12} & \gamma_{21} & \gamma_{30} \\
\gamma_{03} & \gamma_{12} & \gamma_{21}
\end{array}\right)=\left(\begin{array}{ccc}
0 & \frac{\pi}{2} & 0 \\
x & \bar{x} & \bar{y} \\
y & x & \bar{x}
\end{array}\right)
$$

A flat extension $M(2)$ is determined by the requirement that $C \equiv B^{*} M(1)^{-1} B$ is Toeplitz (cf. (2.4) below), and a calculation shows that this is equivalent to

$$
|y|^{2}-|x|^{2}=\frac{\pi^{2}}{8}
$$

Thus $M(1)$ admits flat extensions and $\gamma$ admits 3 -atomic representing measures. To see which of these are supported in the unit disk, we use (1.6) to compute

$$
N \equiv M_{1-z \bar{z}}(2)=\left(\begin{array}{ccc}
\frac{\pi}{2} & -x & -\bar{x} \\
-\bar{x} & \frac{\pi}{4}-\frac{4}{\pi}|x|^{2} & -\frac{2}{\pi}\left(\bar{x}^{2}+x \bar{y}\right) \\
-x & -\frac{2}{\pi}\left(x^{2}+\bar{x} y\right) & \frac{\pi}{4}-\frac{4}{\pi}|x|^{2}
\end{array}\right) .
$$

Theorem 1.1 implies that the 3 -atomic representing measures for $\gamma^{(2)}$ that are supported in the disk correspond to $x$ and $y$ which satisfy (1.7) and $N \geq 0$. For the latter condition we first require $|x|^{2} \leq \frac{\pi^{2}}{24}$ (positivity of the $2 \times 2$ upper left hand corner of $N)$.

Case 1. $|x|^{2}=\frac{\pi}{24}$. In this case, $N \geq 0$ if and only if $y=\frac{-2 x^{2}}{\bar{x}}$, whence $\operatorname{rank} N=1$; thus $\nu[M(2)]$ has $2(=\operatorname{rank} M(1)-\operatorname{rank} N)$ atoms on the unit circle and one atom in the open disk.

Let $\Delta:=32 \pi \operatorname{det} N=\pi^{4}-48 \pi^{2}|x|^{2}+320|x|^{4}-64|x|^{2}|y|^{2}-256 \operatorname{Re} x^{3} \bar{y}$; in the remaining cases, $N \geq 0$ if and only if $|x|^{2}<\frac{\pi^{2}}{24}$ and $\Delta \geq 0$. 
Case 2. $|x|^{2}<\frac{\pi^{2}}{24}$ and $\Delta=0$. In this case, $N \geq 0$ and $\operatorname{rank} N=2$; one atom is on the circle and two atoms are in the open disk.

Case 3. $|x|^{2}<\frac{\pi^{2}}{24}$ and $\Delta>0$. In this case, $N>0$, so all three atoms are in the open disk.

To compute $\nu[M(2)]$ via the Flat Extension Theorem, we note that there is a dependence relation in the column space of the extension $M(3)$ of the form

$$
Z^{3}=\frac{y}{\pi} 1-\frac{4}{\pi^{2}}\left(x^{2}-y \bar{x}\right) Z+\frac{4}{\pi} x Z^{2} ;
$$

the support of $\nu[M(2)]$ consists of the roots of the cubic polynomial

$$
z^{3}-\left(\frac{y}{\pi}-\frac{4}{\pi^{2}}\left(x^{2}-y \bar{x}\right) z+\frac{4}{\pi} x z^{2}\right),
$$

and the densities may be computed from the Vandermonde equation.

As noted above, the Flat Extension Theorem shows that if $M(n)$ admits a flat extension $M(n+1)$, then $M(n+1)$ admits a unique flat extension $M(\infty)$, which in turn determines a unique $\operatorname{rank} M(n)$-atomic representing measure $\nu[M(n+1)]$ corresponding to $M(n+1)$. In the proof of Theorem[1.1, in order to establish that $\operatorname{supp} \nu[M(n+1)] \subseteq K_{p}$, we assume $M_{p}(n+k) \geq 0$ and we need to verify that $M_{p}(\infty) \geq 0$. The following structure theorem for localizing matrices, which is our main technical result, shows that $M_{p}(\infty)$ is positive because it is a flat extension of $M_{p}(n+k)$.

Theorem 1.5. Suppose $M(n+1)$ is a flat extension of $M(n) \geq 0$, and let $p \in$ $\mathbb{C}[z, \bar{z}]$, with $\operatorname{deg} p=2 k$ or $\operatorname{deg} p=2 k-1$. If $M_{p}(n+k) \geq 0$, then for each $d \geq 0$, there is a transition matrix $W \equiv W_{n, d}$ such that

$$
M_{p}(n+k+d+1)=\left(\begin{array}{cc}
M_{p}(n+k+d) & M_{p}(n+k+d) W \\
W^{*} M_{p}(n+k+d) & W^{*} M_{p}(n+k+d) W
\end{array}\right) .
$$

In particular, $M_{p}(n+k+d+1)$ is a flat (positive) extension of $M_{p}(n+k+d)$.

The extension of Theorem 1.1 to general semi-algebraic sets is an immediate consequence of Theorem 1.1 and its proof. Let $\mathcal{P}=\left\{p_{1}, \ldots, p_{m}\right\} \subseteq \mathbb{C}[z, \bar{z}]$ and define $k_{i}$ by $\operatorname{deg} p_{i}=2 k_{i}$ or $\operatorname{deg} p_{i}=2 k_{i}-1(1 \leq i \leq m)$.

Theorem 1.6. There exists a $\operatorname{rank} M(n)$-atomic representing measure for $\gamma^{(2 n)}$ supported in $K_{\mathcal{P}}:=\left\{z \in \mathbb{C}: p_{i}(z, \bar{z}) \geq 0,1 \leq i \leq m\right\}$ if and only if $M(n) \geq 0$ and there is some flat extension $M(n+1)$ for which $M_{p_{i}}\left(n+k_{i}\right) \geq 0$ $(1 \leq i \leq m)$. In this case, the measure $\nu[M(n+1)]$ is a $\operatorname{rank} M(n)$-atomic representing measure supported in $K_{\mathcal{P}}$, with precisely $\operatorname{rank} M(n)-\operatorname{rank} M_{p_{i}}\left(n+k_{i}\right)$ atoms in $\mathcal{Z}\left(p_{i}\right)(1 \leq i \leq m)$.

We illustrate Theorem 1.6 by giving a partial parameterization of the minimal quadrature rules of degree 2 for Lebesgue measure on the triangle with vertices 0 , $1, i$.

Example 1.7. Let $p_{1}(z, \bar{z})=\frac{z+\bar{z}}{2}, p_{2}(z, \bar{z})=\frac{z-\bar{z}}{2 i}, p_{3}(z, \bar{z})=1-\left(\frac{z+\bar{z}}{2}+\frac{z-\bar{z}}{2 i}\right)$; thus $K_{\mathcal{P}} \equiv\left\{z \in \mathbb{C}: p_{i}(z, \bar{z}) \geq 0,1 \leq i \leq 3\right\}$ is the triangle with vertices $0,1, i$. The complex moments up to degree 2 for Lebesgue measure on $K_{\mathcal{P}}$ are $\gamma_{00}=\frac{1}{2}$, $\gamma_{01}=\frac{1+i}{6}, \gamma_{10}=\frac{1-i}{6}, \gamma_{02}=\frac{i}{12}, \gamma_{20}=-\frac{i}{12}, \gamma_{11}=\frac{1}{6}$, and we also have $\gamma_{12} \stackrel{\frac{1}{=}}{=}$ 
$\frac{1+i}{15}$. We seek to characterize the representing measures $\nu$ of least support which interpolate $\gamma_{12}$ as well as $\gamma^{(2)}$ and which satisfy $\operatorname{supp} \nu \subseteq K_{\mathcal{P}}$.

Since $\gamma_{12}$ is prescribed, a calculation shows that a flat extension $M(2)$ of $M(1)$ $(>0)$ is determined by a choice $\gamma_{03}=y_{1}+i y_{2}$ satisfying

$$
\left(y_{1}+\frac{1}{120}\right)^{2}+\left(y_{2}-\frac{1}{120}\right)^{2}=\left(\frac{\sqrt{3}}{120}\right)^{2} \text {. }
$$

Since $\operatorname{deg} p_{i}=1(1 \leq i \leq 3)$, Theorem 1.6 implies that $\nu[M(2)]$ is supported in $K_{\mathcal{P}}$ if and only if $M_{p_{i}}(2) \geq 0(1 \leq i \leq 3)$, and these conditions are equivalent to

$$
\begin{aligned}
& \left(y_{1}+\frac{1}{240}\right)^{2}+\left(y_{2}-\frac{1}{60}\right)^{2} \leq\left(\frac{7}{240}\right)^{2}, \\
& \left(y_{1}+\frac{1}{60}\right)^{2}+\left(y_{2}-\frac{1}{240}\right)^{2} \leq\left(\frac{7}{240}\right)^{2}, \\
& \left(y_{1}-\frac{1}{240}\right)^{2}+\left(y_{2}+\frac{1}{240}\right)^{2} \leq\left(\frac{1}{40 \sqrt{2}}\right)^{2} .
\end{aligned}
$$

The solutions to (1.8)-(1.11) comprise an arc of the circle (1.8). Using the Flat Extension Theorem, we find a dependence relation in the column space of $M(3)$ of the form

$$
Z^{3}=c_{0} 1+c_{1} Z+c_{2} Z^{2}
$$

where

$$
\begin{aligned}
& c_{0}=\frac{2}{25}(1+i)\left(i+(30-30 i) y_{1}+(30+30 i) y_{2}\right), \\
& c_{1}=\frac{1}{50} i\left(-37+(360+360 i) y_{1}-(360-360 i) y_{2}\right), \\
& c_{2}=(-1-i)\left(-1+(6+6 i) y_{1}-(6-6 i) y_{2}\right) ;
\end{aligned}
$$

the support of $\nu[M(2)]$ consists of the roots of $z^{3}-\left(c_{0}+c_{1} z+c_{2} z^{2}\right)$. One solution to (1.8)-(1.11) occurs with $y_{1}=-\frac{1}{120}+\frac{1}{40 \sqrt{6}}$ and $y_{2}=\frac{1}{120}-\frac{1}{40 \sqrt{6}}$; the atoms computed using (1.12) are

$$
\begin{aligned}
& z_{0} \approx 0.155051+0.155051 i, \\
& z_{1} \approx 0.655928+0.166546 i, \\
& z_{2} \approx 0.166546+0.655928 i,
\end{aligned}
$$

with corresponding densities $\rho_{0} \approx 0.152045, \rho_{1}=\rho_{2} \approx 0.173977$.

Theorem 1.6 gives a criterion for the existence of $\operatorname{rank} M(n)$-atomic representing measures supported in $K_{\mathcal{P}}$, but in the general case minimal representing measures have more than $\operatorname{rank} M(n)$ atoms. In [CuFi6] Theorem 1.5] we proved that $\gamma^{(2 n)}$ has a finitely atomic representing measure if and only if there is some $k \geq 0$ such that $M(n)$ admits an extension to a positive moment matrix $M(n+k)$, which in turn admits a flat extension $M(n+k+1)$. It is known that $k>0$ may be required, in which case a minimal representing measure has more than rank $M(n)$ atoms CuFi6, Theorem 5.2]; a concrete example of this phenomenon (for $n=3$ ) appears in [Fi2, Theorem 3.1]. This example is based on [Fi2, Theorem 2.2], which provides an algorithm for computing the minimal representing measure for any moment matrix $M(n)$ in which there is a column relation of the form $Z^{m}=p(Z, \bar{Z})$ for 
some $m, 1 \leq m \leq n$, and some $p \in \mathcal{P}_{m-1}$. Despite this result, relatively little is known about minimal representing measures arising from rank-increasing moment matrix extensions. By combining [CuFi6, Theorem 1.5] with Theorem [1.6] we obtain the following abstract solution to the Truncated Complex $K$-Moment Problem.

Theorem 1.8. $\gamma^{(2 n)}$ admits a finitely atomic representing measure supported in $K_{\mathcal{P}}$ if and only if for some $k \geq 0, M(n)$ admits a positive extension $M(n+k)$ which in turn has a flat extension $M(n+k+1)$ satisfying $M_{p_{i}}\left(n+k+k_{i}\right) \geq 0$ $(1 \leq i \leq m)$.

We conclude this section by comparing Theorem 1.8 to a theorem of Schmüdgen Sch which solves the Full $K$-Moment Problem on $\mathbb{R}^{n}$ in the case when $K \equiv K_{\mathcal{P}}$ is a compact semi-algebraic set. Schmüdgen's result is actually stated in terms of positive sequences, but for $\mathbb{R}^{2}$ we can complexify Schmüdgen's result and paraphrase it in the language of moment matrices as follows: A full moment sequence $\left(\gamma_{i j}\right)_{i, j \geq 0}$ $\left(\gamma_{00}>0, \gamma_{i j}=\bar{\gamma}_{j i}\right)$ has a representing measure supported in a compact semialgebraic set $K_{\mathcal{P}}$ if and only if $M(\infty)(\gamma) \geq 0$ and $M_{q}(\infty)(\gamma) \geq 0$ for every polynomial $q$ that is a product of distinct $p_{i}$ 's. Thus, in the case when the semialgebraic set is compact, the sole criterion for representing measures is positivity of moment matrices and localizing matrices. By contrast, Theorem 1.8 applies to any semi-algebraic set and entails positivity only for the $M_{p_{i}}$ matrices (not the $M_{q}$ 's); however, Theorem 1.8 presupposes the existence of certain positive and flat extensions $M(n+k)$ and $M(n+k+1)$.

Acknowledgements. This research commenced during Fall 1996, while the secondnamed author was visiting The University of Iowa on a sabbatical leave; he is grateful to the Department of Mathematics for its hospitality and support. Many of the examples and some of the theorems in this paper were proved using calculations with the software tool Mathematica.

\section{Flat EXtensions of POSitive moment MATRiCES}

Given $\gamma \equiv \gamma^{(2 n)}$, for $0 \leq i, j \leq n$ we define the $(i+1) \times(j+1)$ matrix $B_{i j}$ whose entries are the moments of order $i+j$ :

$$
B_{i j}:=\left(\begin{array}{ccccc}
\gamma_{i j} & \gamma_{i+1, j-1} & & \cdots & \gamma_{i+j, 0} \\
\gamma_{i-1, j+1} & \gamma_{i j} & \gamma_{i+1, j-1} & & \\
& \gamma_{i-1, j+1} & & & \vdots \\
\vdots & & & & \\
& & & & \\
\gamma_{0, j+i} & & & \cdots & \gamma_{j i}
\end{array}\right) .
$$

It follows from equation (1.3) that $M(n)(\gamma)$ admits a block decomposition $M(n)=\left(B_{i j}\right)_{0 \leq i, j \leq n}$.

We may also define blocks $B_{0, n+1}, \ldots, B_{n-1, n+1}$ via (2.1). Given "new moments" of degree $2 n+1$ for a prospective representing measure, let $B_{n, n+1}$ denote the corresponding moment matrix block given by (2.1), and let

$$
B(n+1):=\left(\begin{array}{c}
B_{0, n+1} \\
\vdots \\
B_{n-1, n+1} \\
B_{n, n+1}
\end{array}\right) .
$$


Given a moment matrix block $C(n+1)$ of the form $B_{n+1, n+1}$ (corresponding to "new moments" of degree $2 n+2)$, we may describe the moment matrix extension $M(n+1)$ via the block decomposition

$$
M(n+1)=\left(\begin{array}{cc}
M(n) & B(n+1) \\
B(n+1)^{*} & C(n+1)
\end{array}\right) .
$$

A theorem of Smul'jan $\mathrm{Smu}$ shows that a block matrix

$$
M=\left(\begin{array}{cc}
A & B \\
B^{*} & C
\end{array}\right)
$$

is positive if and only if (i) $A \geq 0$, (ii) there exists a matrix $W$ such that $B=A W$, and (iii) $C \geq W^{*} A W$ (since $A=A^{*}, W^{*} A W$ is independent of $W$ provided $B=$ $A W)$. Note also that if $M \geq 0$, then $\operatorname{rank} M=\operatorname{rank} A$ if and only if $C=W^{*} A W$; conversely, if $A \geq 0$ and there exists $W$ such that $B=A W$ and $C=W^{*} A W$, then $M \geq 0$ and $\operatorname{rank} M=\operatorname{rank} A$. A block matrix $M$ as in (2.3) is an extension of $A$, and is a flat extension if $\operatorname{rank} M=\operatorname{rank} A$. A flat extension of a positive matrix $A$ is completely determined by a choice of block $B$ satisfying $B=A W$ and $C=W^{*} A W$ for some matrix $W$; we denote such a flat extension by $[A ; B]$.

For an $(n+1) \times(n+2)$ matrix $B_{n, n+1}$, representing "new moments" of degree $2 n+1$ for a prospective representing measure of $\gamma^{(2 n)}$, let

$$
B:=\left(\begin{array}{c}
B_{0, n+1} \\
\vdots \\
B_{n-1, n+1} \\
B_{n, n+1}
\end{array}\right) .
$$

By Smul'jan's theorem, $M(n) \geq 0$ admits a (necessarily positive) flat extension

$$
[M(n) ; B]=\left(\begin{array}{cc}
M(n) & B \\
B^{*} & C
\end{array}\right)
$$

in the form of a moment matrix $M(n+1)(c f .(2.2))$ if and only if

$$
\begin{aligned}
& B=M(n) W \text { for some } W \text { (i.e., } \operatorname{Ran} B \subseteq \operatorname{Ran} M(n)) ; \\
& C \equiv W^{*} M(n) W \text { is Toeplitz, i.e., has the form of a moment matrix } \\
& \text { block } B_{n+1, n+1} .
\end{aligned}
$$

Theorem 2.1 (Flat Extension Theorem). (cf. CuFi4 Remark 3.15, Theorem 5.4, Corollary 5.12, Theorem 5.13, and Corollary 5.15], [CuFi5, Lemma 1.9], Fi3) Suppose $M(n)(\gamma)$ is positive and admits a flat extension $M(n+1)$, so that $Z^{n+1}=$ $p(Z, \bar{Z})$ in $\mathcal{C}_{M(n+1)}$ for some $p \in \mathcal{P}_{n}$. Then there exist unique successive flat (positive, recursively generated) moment matrix extensions $M(n+2), M(n+3)$, ..., which are determined by the relations

$$
Z^{n+k}=\left(z^{k-1} p\right)(Z, \bar{Z}) \in \mathcal{C}_{M(n+k)} \quad(k \geq 2) .
$$

Let $r:=\operatorname{rank} M(n)$. There exist unique scalars $a_{0}, \ldots, a_{r-1}$ such that in $\mathcal{C}_{M(r)}$,

$$
Z^{r}=a_{0} 1+\cdots+a_{r-1} Z^{r-1} .
$$

The characteristic polynomial $g_{\gamma}(z) \equiv z^{r}-\left(a_{0}+\cdots+a_{r-1} z^{r-1}\right)$ has $r$ distinct roots, $z_{0}, \ldots, z_{r-1}$, and $\gamma$ has a rank $M(n)$-atomic minimal representing measure of the form

$$
\nu=\nu[M(n+1)]=\sum \rho_{i} \delta_{z_{i}}
$$


where the densities $\rho_{i}>0$ are determined by the Vandermonde equation

$$
V\left(z_{0}, \ldots, z_{r-1}\right)\left(\rho_{0}, \ldots, \rho_{r-1}\right)^{t}=\left(\gamma_{00}, \ldots, \gamma_{0, r-1}\right)^{t} .
$$

The measure $\nu[M(n+1)]$ is the unique representing measure for $\gamma^{(2 n+2)}$, and is also the unique representing measure for $M(\infty)$.

We note in connection with (2.5) that due to the structure of moment matrix blocks $B_{i j}$, an extension $M(n+1)$ is completely determined from $M(n)$ once column $Z^{n+1}$ is specified.

Finally, we recall from [CuFi4], [Fi1] that $M(n) \geq 0$ is recursively generated if the following property holds:

$$
p, q, p q \in \mathcal{P}_{n}, p(Z, \bar{Z})=0 \quad \Longrightarrow \quad(p q)(Z, \bar{Z})=0 .
$$

If $M(n) \geq 0$ admits a flat extension $M(n+1)$, then $M(n+1)$, and all of its successive flat extensions $M(n+1+d)$ (described by Theorem[2.1), are recursively generated [CuFi4, Remark 3.15-ii)].

\section{LOCALIZING MATRICES}

Given $\gamma \equiv \gamma^{(2 n)}, k \leq n$, and $p \in \mathcal{P}_{2 k}$, we define a localizing matrix $M_{p}(n) \equiv$ $M_{p}(n)(\gamma)$, whose positivity is a necessary condition for the existence of a representing measure for $\gamma$ supported in $K_{p}:=\{z \in \mathbb{C}: p(z, \bar{z}) \geq 0\}$. The purpose of this section is to prove Theorem 1.2 (restated as Theorem 3.5 below), which shows how to compute $M_{p}(n)$ as a linear combination of compressions of $M(n)$. In the next section, this description of $M_{p}(n)$ will be utilized to characterize the existence of minimal representing measures supported in a prescribed semi-algebraic set.

Motivated by Riesz's analysis of moment problems in $\mathbb{R}$ [kkh, we define the Riesz linear functional $\Lambda_{\gamma}: \mathcal{P}_{2 n} \rightarrow \mathbb{C}$ by

$$
\Lambda_{\gamma}\left(\sum_{0 \leq r+s \leq 2 n} a_{r s} \bar{z}^{r} z^{s}\right):=\sum_{0 \leq r+s \leq 2 n} a_{r s} \gamma_{r s} .
$$

Recall from [CuFi4] that $M(n) \equiv M(n)(\gamma)$ may be defined as follows: for $0 \leq$ $i+j \leq n, 0 \leq k+l \leq n$,

$$
\left\langle M(n) \widehat{\bar{z}^{i} z^{j}}, \widehat{\bar{z}^{k} z^{l}}\right\rangle:=\gamma_{i+l, j+k}
$$

thus

$$
\begin{aligned}
\left\langle M(n) \widehat{\bar{z}^{i} z^{j}}, \widehat{\bar{z}^{k} z^{l}}\right\rangle & =\Lambda_{\gamma}\left(\bar{z}^{i+l} z^{j+k}\right) \\
& =\Lambda_{\gamma}\left(\left(\bar{z}^{i} z^{j}\right)\left(\overline{\bar{z}^{k} z^{l}}\right)\right),
\end{aligned}
$$

whence, by linearity,

$$
\langle M(n) \hat{f}, \hat{g}\rangle=\Lambda_{\gamma}(f \bar{g}) \quad\left(f, g \in \mathcal{P}_{n}\right) .
$$

Let $p \in \mathcal{P}_{2 n}, \operatorname{deg} p=2 k$ or $\operatorname{deg} p=2 k-1(0 \leq k \leq n)$, and set $\sigma \equiv \sigma(k, n):=$ $\frac{(n-k+1)(n-k+2)}{2}$. The map

$$
\varphi_{p}: \mathbb{C}^{\sigma} \times \mathbb{C}^{\sigma} \rightarrow \mathbb{C}
$$

defined by

$$
\varphi_{p}(\hat{f}, \hat{g})=\Lambda_{\gamma}(p f \bar{g}) \quad\left(f, g \in \mathcal{P}_{n-k}\right)
$$


is sesquilinear; thus, by the Riesz representation theorem for sesquilinear forms, there exists a unique matrix $M_{p}(n) \equiv M_{p}(n)(\gamma) \in M_{\sigma}(\mathbb{C})$ (which we sometimes abbreviate as $M_{p}$ ) such that

$$
\varphi_{p}(\hat{f}, \hat{g})=\left(M_{p}(n) \hat{f}, \hat{g}\right) \quad\left(=\Lambda_{\gamma}(p f \bar{g})\right) \quad\left(f, g \in \mathcal{P}_{n-k}\right) .
$$

Note that if there exists a representing measure $\mu$ for $\gamma$ supported in $K_{p}$, then

$$
\left(M_{p} \hat{f}, \hat{f}\right)=\Lambda_{\gamma}\left(p|f|^{2}\right)=\int p|f|^{2} d \mu \geq 0 \quad\left(f \in \mathcal{P}_{n-k}\right),
$$

whence $M_{p} \geq 0$.

Our first goal is to describe $M_{p}(n)$ concretely, as a linear combination of certain compressions of $M(n)$. Note that if $r, s \geq 0,0 \leq r+s \leq 2 k$, then there exist $i, j \geq 0$ such that

$$
\bar{z}^{r} z^{s}=\bar{z}^{i} z^{j} \cdot \bar{z}^{r-i} z^{s-j} \quad(0 \leq i+j \leq k, 0 \leq(r+s)-(i+j) \leq k) .
$$

(In the sequel, we abbreviate these conditions by writing $0 \leq i+j,(r+s)-(i+j) \leq$ $k$.) Indeed, $(i, j)$ can be chosen as the integral point in the triangle $0 \leq u+v \leq k$ closest to $(r, s)$.

Lemma 3.1. Let $0 \leq k \leq n, 0 \leq r+s \leq 2 k$, and suppose $p(z, \bar{z}) \equiv \bar{z}^{r} z^{s}=$ $\bar{z}^{i} z^{j} \cdot \bar{z}^{r-i} z^{s-j}$ with $0 \leq i+j,(r-i)+(s-j) \leq k$. For $f, g \in \mathcal{P}_{n-k}$,

$$
\left\langle M_{p}(n) \hat{f}, \hat{g}\right\rangle=\left\langle M(n) \widehat{\bar{z}^{i} z^{j} f},\left(z^{r-i} \bar{z}^{s-j} g\right) \hat{\rangle}\right. \text {. }
$$

Proof.

$$
\begin{aligned}
& \left\langle M_{p}(n) \hat{f}, \hat{g}\right\rangle=\Lambda_{\gamma}(p f \bar{g}) \\
& =\Lambda_{\gamma}\left(\left(\bar{z}^{i} z^{j} f\right) \overline{\left(z^{r-i} \bar{z}^{s-j} g\right)}\right) \quad(\text { with } 0 \leq i+j+n-k, r-i+s-j+n-k \leq n) \\
& =\left\langle M(n) \overline{\bar{z}^{i} z^{j} f},\left(z^{r-i} \bar{z}^{s-j} g\right)\right\rangle .
\end{aligned}
$$

Proposition 3.2. Let $0 \leq k \leq n$. For $0 \leq r+s \leq 2 k, 0 \leq t+u, q+v \leq n-k$,

$$
\left\langle M_{\bar{z}^{r} z^{s}}(n) \widehat{\bar{z}^{q} z^{v}}, \widehat{\bar{z}^{t} z^{u}}\right\rangle=\gamma_{r+q+u, s+v+t}
$$

Proof. Write $\bar{z}^{r} z^{s}=\bar{z}^{i} z^{j} \cdot \bar{z}^{r-i} z^{s-j}$ with $0 \leq i+j,(r-i)+(s-j) \leq k$. Lemma 3.1 implies

$$
\begin{aligned}
\left\langle M_{\bar{z}^{r} z^{s}}(n) \widehat{\bar{z}^{q} z^{v}}, \widehat{\bar{z}^{t} z^{u}}\right\rangle & =\left\langle M(n)\left(\bar{z}^{i} z^{j} \bar{z}^{q} z^{v} \widehat{)},\left(z^{r-i} \bar{z}^{s-j} \bar{z}^{t} z^{u}\right)^{\uparrow}\right\rangle\right. \\
& =\left\langle M(n)\left(\bar{z}^{i+q} z^{j+v}\right) \widehat{,},\left(\bar{z}^{s+t-j} z^{r+u-i}\right)^{\uparrow}\right\rangle \\
& =\gamma_{(i+q)+(r+u-i),(j+v)+(s+t-j)} \\
& =\gamma_{r+q+u, s+v+t} .
\end{aligned}
$$

Note that in Lemma 3.1 and Proposition 3.2 we are not assuming that $p(z, \bar{z}) \equiv$ $\bar{z}^{r} z^{s}$ satisfies $\operatorname{deg} p=2 k$ or $\operatorname{deg} p=2 k-1$, only that $\operatorname{deg} p \leq 2 k$; thus the size of $M_{p}(n)$ may be greater than $\frac{(n-k+1)(n-k+2)}{2}$ and the vectors $\hat{f}, \hat{g}$ are then defined with respect to that larger size. We consider this more general framework because in the sequel $\bar{z}^{r} z^{s}$ may contribute to a polynomial $q$ with $\operatorname{deg} q>r+s$.

Let $p, q, t, u \geq 0$ and suppose $A$ is a matrix whose columns are indexed by

$$
\bar{Z}^{0} Z^{p}, \ldots, \bar{Z}^{p} Z^{0}, \ldots, \bar{Z}^{0} Z^{p+q}, \ldots, \bar{Z}^{p+q} Z^{0},
$$


and whose rows are indexed by

$$
\bar{Z}^{0} Z^{t}, \ldots, \bar{Z}^{t} Z^{0}, \ldots, \bar{Z}^{0} Z^{t+u}, \ldots, \bar{Z}^{t+u} Z^{0} .
$$

Suppose $0 \leq r+s \leq p+q, r, s \geq 0$. The columns of $A$ that are indexed by multiples of $\bar{Z}^{r} Z^{s}$ are of the form

$$
\bar{Z}^{r+i} Z^{s+j}, \quad \max (0, p-(r+s)) \leq i+j \leq(p+q)-(r+s) .
$$

Let $\kappa$ denote the number of such columns; if $p \geq r+s$,

$$
\begin{aligned}
\kappa & =p-(r+s)+1+\cdots+(p+q)-(r+s)+1 \\
& =(q+1)\left(p+\frac{q}{2}+1-(r+s)\right) ;
\end{aligned}
$$

if $p \leq r+s$,

$$
\begin{aligned}
\kappa & =1+\cdots+(p+q)-(r+s)+1 \\
& =\frac{((p+q)-(r+s)+1)(p+q-(r+s)+2)}{2} .
\end{aligned}
$$

For $\kappa \geq \sigma \geq 1$, we denote the compression of $A$ to the first $\sigma$ of these columns corresponding to total degree at least $d$ by $A_{\left[\bar{Z}^{r} Z^{s}\right]} \equiv A_{\left[\bar{Z}^{r} Z^{s} ; d, \sigma\right]}$ (assuming that there are at least $\sigma$ such columns). Similarly, let $\tau$ denote the number of rows of $A$ indexed by multiples of $\bar{Z}^{m} Z^{l}$, and for $1 \leq \rho \leq \tau$, let ${ }_{\left[\bar{Z}^{m} Z^{l}\right]} A \equiv{ }_{\left[\bar{Z}^{m} Z^{l} ; e, \rho\right]} A$ denote the compression of $A$ to the first $\rho$ of these rows corresponding to total degree at least $e$ (assuming there are at least $\rho$ such rows). We denote the compression of $A$


values of $e, \rho, d, \sigma$ are clear from the context.

We omit the proof of the following elementary result.

\section{Lemma 3.3.}

(i) ${ }_{[\cdot]} A_{[\cdot]}=\left({ }_{[\cdot]} A\right)_{[\cdot]}={ }_{[\cdot]}\left(A_{[\cdot]}\right)$;

(ii) $(A B)_{[\cdot]}=A\left(B_{[\cdot]}\right) ;[\cdot](A B)=\left({ }_{[\cdot]} A\right) B$;

(iii) ${ }_{[\cdot]}(A B)_{[\cdot]}=\left({ }_{[\cdot]} A\right)\left(B_{[\cdot]}\right)$;

(iv) $\left({ }_{\left[\bar{Z}^{m} Z^{l} ; e, \rho\right]}^{A}\right)^{*}=A_{\left[\bar{Z}^{m} Z^{l} ; e, \rho\right]}^{*}$ (here, the convention is that if the rows of $A$ are indexed by $Z^{p}, Z^{p-1} \bar{Z}, \ldots$, the columns of $A^{*}$ are also indexed by $Z^{p}, Z^{p-1} \bar{Z}, \ldots$, and similarly for the columns of $A$ and the rows of $\left.A^{*}\right)$.

Lemma 3.4. Suppose $0 \leq k \leq n$ and let $\sigma=\frac{(n-k+1)(n-k+2)}{2}$. For $0 \leq p+q$, $l+m \leq k$, let $M \equiv{ }_{\left[\bar{Z}^{m} Z^{l} ; 0, \sigma\right]}{ }^{M(n)}{ }_{\left[\bar{Z}^{p} Z^{q} ; 0, \sigma\right]}$, the compression of $M(n)$ to the first $\sigma$ columns indexed by multiples of $\bar{Z}^{p} Z^{q}$ and to the first $\sigma$ rows indexed by multiples of $\bar{Z}^{m} Z^{l}$. Then $M=\left[M_{\bar{z}^{p} z^{q} \cdot \bar{z}^{l} z^{m}}(n)\right]_{\sigma}$, the compression of $M_{\bar{z}^{p} z^{q} \cdot \bar{z}^{l} z^{m}}(n)$ to its first $\sigma$ rows and columns.

Proof. The columns of $M$ are indexed by $\bar{Z}^{p+i} Z^{q+j}(0 \leq i+j \leq n-k)$ and the rows are indexed by $\bar{Z}^{m+a} Z^{l+b}(0 \leq a+b \leq n-k)$. The entry in row $\bar{Z}^{m+a} Z^{l+b}$, column $\bar{Z}^{p+i} Z^{q+j}$ of $M$ is thus $\gamma_{p+i+l+b, q+j+m+a}$. The corresponding entry of $M_{\bar{z}^{p} z^{q} \cdot \bar{z}^{l} z^{m}}(n)$, located in row $\bar{Z}^{a} Z^{b}$, column $\bar{Z}^{i} Z^{j}$, is $\left\langle M_{\bar{z}^{p} z^{q} \cdot \bar{z}^{l} z^{m}}(n) \widehat{\bar{z}^{i} z^{j}}, \widehat{\bar{z}^{a} z^{b}}\right\rangle=$ 
$\left\langle M_{\bar{z}^{p+l} z^{q+m}}(n) \widehat{\bar{z}^{i} z^{j}}, \widehat{\bar{z}^{a} z^{b}}\right\rangle=\gamma_{p+l+i+b, q+m+j+a}$ (by Proposition 3.2 since $p+l+$ $q+m \leq 2 k)$, so the result follows.

The main result of this section, which follows, describes $M_{p}(n)$ in terms of compressions of $M(n)$. Let $0 \leq k \leq n$ and suppose $p(z, \bar{z}) \equiv \sum_{0 \leq r+s \leq d} a_{r s} \bar{z}^{r} z^{s}$, with $d \equiv \operatorname{deg} p=2 k$ or $2 k-1$.

Recall that for each $r, s \geq 0$ with $0 \leq r+s \leq 2 k$, there exist $i \equiv i(r, s, k)$, $j \equiv j(r, s, k), t \equiv t(r, s, k), u \equiv u(r, s, k) \geq 0$ such that $i+j, t+u \leq k, i+t=r$, $j+u=s ;$ thus

$$
p(z, \bar{z})=\sum_{0 \leq r+s \leq d} a_{r s} \bar{z}^{i} z^{j} \cdot \bar{z}^{r-i} z^{s-j} .
$$

Since $\operatorname{deg} p=2 k$ or $2 k-1$, the size of $M_{p}(n)$ is $\sigma \times \sigma$, where $\sigma=\frac{(n-k+1)(n-k+2)}{2}$. Thus, by (3.2) and the uniqueness property of $M_{p}(n)$,

$$
M_{p}(n)=\sum a_{r s}\left[M_{\bar{z}^{r} z^{s}}(n)\right]_{\sigma}
$$

and Lemma 3.4 implies that for each $r, s$,

$$
\begin{aligned}
& {\left[M_{\bar{z}^{r} z^{s}}(n)\right]_{\sigma}=\left[M_{\bar{z}^{i} z^{j} \cdot \bar{z}^{r-i} z^{s-j}}(n)\right]_{\sigma}} \\
& \left.=\left[\bar{Z}^{s-j} Z^{r-i} ; 0, \sigma\right]\right]^{M(n)}\left[\bar{Z}^{i} Z^{j} ; 0, \sigma\right] \cdot
\end{aligned}
$$

Note also from Proposition 3.2 and the proof of Lemma 3.4 that we may obtain $\left[M_{\bar{z}^{r} z^{s}}(n)\right]_{\sigma}$ from $M(n-k)$ by replacing each $\gamma_{i j}$ in the latter matrix by $\gamma_{r+i, s+j}$.

We thus have the following computational description of $M_{p}(n)$, which restates and refines Theorem 1.2 .

\section{Theorem 3.5.}

(i) $M_{p}(n)=\sum_{0 \leq r+s \leq d} a_{r s\left[\bar{Z}^{s-j} Z^{r-i} ; 0, \sigma\right]} M(n)_{\left[\bar{Z}^{i} Z^{j} ; 0, \sigma\right]} \cdot$

(ii) The entries in $M_{p}(n)$ are of the form $\sum_{0 \leq r+s \leq d} a_{r s} \gamma_{r+u, s+v}(0 \leq u+v \leq$ $2(n-k))$.

We note that the construction of $M_{p}(n)$ in Theorem 3.5)(ii) is independent of the way in which we factor the monomials of $p$ as in (3.4), as the following example shows.

Example 3.6. Let $n=3, p(z, \bar{z})=z+z^{2} \bar{z}, k=2$; then $\operatorname{deg} p=2 k-1$. Consider the decompositions

$$
p_{1}=z \cdot 1+z^{2} \cdot \bar{z} \quad \text { and } \quad p_{2}=1 \cdot z+\bar{z} z \cdot z
$$

we have $\sigma=\frac{(n-k+1)(n-k+2)}{2}=3$. Now

$$
\begin{aligned}
& \left.M_{p_{1}}(3)=\left[\bar{Z}^{0} Z^{0} ; 0,3\right]\right]^{M(3)_{\left[\bar{Z}^{0} Z ; 0,3\right]}}+{ }_{\left[\bar{Z}^{0} Z^{1} ; 0,3\right]} M(3)_{\left[Z^{2} \bar{Z}^{0} ; 0,3\right]} \\
& Z \quad Z^{2} \quad Z \bar{Z} \quad Z^{2} \quad Z^{3} \quad Z^{2} \bar{Z} \\
& =\frac{1}{\bar{Z}}\left(\begin{array}{lll}
\gamma_{01} & \gamma_{02} & \gamma_{11} \\
\gamma_{11} & \gamma_{12} & \gamma_{21} \\
\gamma_{02} & \gamma_{03} & \gamma_{12}
\end{array}\right)+{ }_{Z \bar{Z}}^{Z}{ }_{Z \bar{Z}}\left(\begin{array}{ccc}
\gamma_{12} & \gamma_{13} & \gamma_{22} \\
\gamma_{22} & \gamma_{23} & \gamma_{32} \\
\gamma_{13} & \gamma_{14} & \gamma_{23}
\end{array}\right),
\end{aligned}
$$


and, similarly,

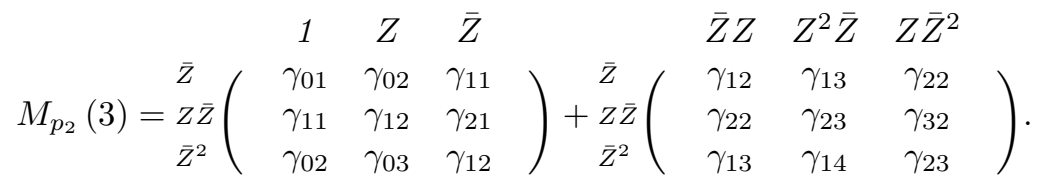

We next present some properties of $M_{p}(n)$.

\section{Proposition 3.7.}

(i) For $p \equiv 1, M_{p}(n)=M(n)$.

(ii) For $1 \leq k \leq n, f \in \mathcal{P}_{k}, p=|f|^{2}, h, g \in \mathcal{P}_{n-k}$,

$$
\left\langle M_{|f|^{2}} \hat{g}, \hat{h}\right\rangle=\langle M(n) \widehat{f g}, \widehat{f h}\rangle \text {. }
$$

(iii) For $k=n, \operatorname{deg} f=n, p=|f|^{2}, M_{p}(n)=(\langle M(n) \hat{f}, \hat{f}\rangle)$.

(iv) Let $0 \leq k \leq n$ and let $p=f_{1} \bar{f}_{2}$, with $f_{1}, f_{2} \in \mathcal{P}_{k}$. For $g_{1}, g_{2} \in \mathcal{P}_{n-k}$,

$$
\left\langle M_{p} \hat{g}_{1}, \hat{g}_{2}\right\rangle=\left\langle M(n) \widehat{f_{1} g_{1}}, \widehat{f_{2} g_{2}}\right\rangle \text {. }
$$

Proof. (ii) Clear.

(ii) $\left\langle M_{|f|^{2}} \hat{g}, \hat{h}\right\rangle=\Lambda_{\gamma}\left(|f|^{2} g \bar{h}\right)=\Lambda_{\gamma}(f g(\overline{f h}))=\langle M(n) \widehat{f g}, \widehat{f h}\rangle$.

(iii) For $g, h \in \mathcal{P}_{0}, g \bar{h}\left\langle M_{p}(n) \hat{1}, \hat{1}\right\rangle=\left\langle M_{p}(n) \hat{g}, \hat{h}\right\rangle=\Lambda_{\gamma}(p g \bar{h})$; with $g=h \equiv 1$,

$$
\left\langle M_{p}(n) \hat{1}, \hat{1}\right\rangle=\Lambda_{\gamma}(p)=\Lambda_{\gamma}\left(|f|^{2}\right)=\langle M(n) \hat{f}, \hat{f}\rangle .
$$

(iv) $\left\langle M_{p} \hat{g}_{1}, \hat{g}_{2}\right\rangle=\Lambda_{\gamma}\left(f_{1} \bar{f}_{2} g_{1} \bar{g}_{2}\right)=\Lambda_{\gamma}\left(f_{1} g_{1}\left(\overline{f_{2} g_{2}}\right)\right)=\left\langle M(n) \widehat{f_{1} g_{1}}, \widehat{f_{2} g_{2}}\right\rangle$.

Proposition 3.8. $M_{p}(n)^{*}=M_{\bar{p}}(n)$.

Proof. $\left\langle M_{\bar{p}}(n) \hat{f}, \hat{g}\right\rangle=\Lambda_{\gamma}(\bar{p} f \bar{g})=\overline{\Lambda_{\gamma}(p g \bar{f})}=\overline{\left\langle M_{p}(n) \hat{g}, \hat{f}\right\rangle}=\left\langle\hat{f}, M_{p}(n) \hat{g}\right\rangle=$ $\left\langle M_{p}(n)^{*} \hat{f}, \hat{g}\right\rangle$.

Proposition 3.9. Let $p \in \mathcal{P}_{n}$.

(i) If $n$ is even, or if $n$ is odd and $\operatorname{deg} p<n$, then $M_{p}(n)=0 \Rightarrow p(Z, \bar{Z})=0$ in $\mathcal{C}_{M(n)}$.

(ii) If $\operatorname{deg} p=n$, or if $n$ is even and $\operatorname{deg} p=n-1$, then $p(Z, \bar{Z})=0$ in $\mathcal{C}_{M(n)} \Rightarrow$ $M_{p}(n)=0$.

(iii) If $M(n) \geq 0$ is recursively generated, and $p(Z, \bar{Z})=0$ in $\mathcal{C}_{M(n)}$, then $M_{p}(n)=0$.

Before proving Proposition 3.9, we pause briefly to note that the formulation is sharp in the following sense. Concerning (ii), if $n$ is odd and $\operatorname{deg} p=n$, then we may have $M_{p}(n)=0$ and $p(Z, \bar{Z}) \neq 0$. To see this, consider $n=1, p(z, \bar{z})=z$, $k=1$; here, $M_{z}(1)=\left(\gamma_{01}\right)$ and $Z=\left(\begin{array}{lll}\gamma_{01} & \gamma_{11} & \gamma_{02}\end{array}\right)^{t}$. Concerning (iii), let $n=3$, $p(z, \bar{z})=z^{2}, k=1$, so $\operatorname{deg} p=n-1$; note that $\left(M_{z^{2}}(3) \widehat{z^{2}}, \widehat{z^{2}}\right)=\gamma_{24}$ and $Z^{2}=\left(\gamma_{02}, \gamma_{12}, \gamma_{03}, \gamma_{22}, \gamma_{13}, \gamma_{04}, \gamma_{32}, \gamma_{23}, \gamma_{14}, \gamma_{05}\right)^{t}$, so $Z^{2}=0$ in $\mathcal{C}_{M(3)}$ need not imply $M_{z^{2}}=0$. 
Proof of Proposition 3.9 (1) Let $d \equiv \operatorname{deg} p=2 k$ or $2 k-1$, and denote $p$ as $p(z, \bar{z}) \equiv \sum_{0 \leq r+s \leq d} a_{r s} \bar{z}^{r} z^{s}$. The elements of $M_{p}(n)$ are indexed as $\sum a_{r s} \gamma_{r+u, s+v}$ $(0 \leq u+v \leq 2(n-k))$ (cf. Theorem 3.5](iii)). The components of $p(Z, \bar{Z})$ are of the form $\left\langle p(Z, \bar{Z}), \widehat{\bar{z}^{i} z^{j}}\right\rangle=\sum a_{r s}\left\langle\bar{Z}^{r} Z^{s}, \widehat{\bar{z}^{i} z^{j}}\right\rangle=\sum a_{r s}\left\langle M(n) \widehat{\bar{z}^{r} z^{s}}, \widehat{\bar{z}^{i} z^{j}}\right\rangle=$ $\sum a_{r s} \gamma_{r+j, s+i}(0 \leq i+j \leq n)$. Since $M_{p}(n)=0$, to prove $p(Z, \bar{Z})=0$ it suffices to prove $n \leq 2(n-k)$, i.e., $2 k \leq n$. Since $\operatorname{deg} p \leq n$, we may assume $\operatorname{deg} p=2 k-1$. If $n$ is even, say $n=2 m$, then since $2 k-1=\operatorname{deg} p \leq n=2 m$, it follows that $k \leq m$, whence $2 k \leq n$. If $n$ is odd, i.e., $n=2 m-1$, then $\operatorname{deg} p<n \Rightarrow 2 k-1<2 m-1 \Rightarrow 2 k \leq 2 m-1=n$.

(iii) We use the notation introduced in the proof of (ii). To prove $p(Z, \bar{Z})=0 \Rightarrow$ $M_{p}(n)=0$, it thus suffices to check that $2(n-k) \leq n$ (or $n \leq 2 k$ ) under the conditions of the hypothesis. If $\operatorname{deg} p=n$, the result is clear since $\operatorname{deg} p \leq 2 k$. If $n$ is even, $n=2 m$, and $\operatorname{deg} p=n-1$, then $2 k-1=\operatorname{deg} p=2 m-1$, so $k=m$ and $n=2 m=2 k$.

(iii) We retain the notation introduced above; in particular, the entries of $M_{p}(n)$ are $\sum a_{r s} \gamma_{r+u, s+v}(0 \leq u+v \leq 2(n-k))$. Since $p(Z, \bar{Z})=0$ and $M(n)$ is recursively generated, then $\left(\bar{z}^{l} z^{m} p\right)(Z, \bar{Z})=0(0 \leq l+m \leq n-\operatorname{deg} p)$. Thus, for $0 \leq i+j \leq n, 0=\left\langle\left(\bar{z}^{l} z^{m} p\right)(Z, \bar{Z}), \widehat{\bar{z}^{i} z^{j}}\right\rangle=\left\langle\sum a_{r s} \bar{Z}^{r+l} Z^{s+m}, \widehat{\bar{z}^{i} z^{j}}\right\rangle=$ $\sum a_{r s} \gamma_{r+l+j, s+m+i}$, and it follows that $0=\sum a_{r s} \gamma_{r+a, s+b}(0 \leq a+b \leq 2 n-\operatorname{deg} p)$.

To complete the proof, it suffices to verify that $2(n-k) \leq 2 n-\operatorname{deg} p$, and this follows since $\operatorname{deg} p \leq 2 k$.

Proposition 3.10. Let $\operatorname{deg} p \leq n$ and suppose $\mu$ is a representing measure for $\gamma^{(2 n)}$.

(i) $\operatorname{supp} \mu \subseteq \mathcal{Z}(p) \Rightarrow M_{p}(n)=0$.

(ii) If $n$ is even, or if $n$ is odd and $\operatorname{deg} p<n$, then $M_{p}(n)=0 \Rightarrow \operatorname{supp} \mu \subseteq \mathcal{Z}(p)$.

Proof. (ii) Since $\operatorname{supp} \mu \subseteq \mathcal{Z}(p)$, [CuFi4, Proposition 3.1] implies that $p(Z, \bar{Z})=0$, and [CuFi4, Remark 3.15] implies that $M(n)$ is positive and recursively generated. Proposition 3.9 (iii) thus implies $M_{p}(n)=0$.

(ii) Proposition 3.9(i) implies that $p(Z, \bar{Z})=0$, so the result follows from [CuFi4 Proposition 3.1].

We remark that if $n$ is odd and $\operatorname{deg} p=n$, we may have $M_{p}(n)=0$, but $\operatorname{supp} \mu$ need not be contained in $\mathcal{Z}(p)$; to see this, let $n=1, M(1)=\left(\begin{array}{ccc}1 & 0 & 0 \\ 0 & 1 & 0 \\ 0 & 0 & 1\end{array}\right), p(z, \bar{z})=z$, $k=1$. From [CuFi4 Theorem 6.1], $M(1)$ admits a representing measure $\mu$, and $\operatorname{supp} \mu \nsubseteq \mathcal{Z}(p)=\{0\}$ since $\operatorname{card} \operatorname{supp} \mu \geq \operatorname{rank} M(1)=3$; on the other hand, $M_{p}(1)=\left(\gamma_{01}\right)=0$.

Proposition 3.11. Suppose $\gamma \equiv \gamma^{(2 n)}$ has a representing measure $\mu$ and let $K=$ $\operatorname{supp} \mu$. If $k \leq \frac{n}{2}$ and $p \in \mathcal{P}_{2 k}$, then $M_{p}(n)=0$ if and only if $\left.p\right|_{K}=0$.

Proof. If $\left.p\right|_{K}=0$, then $K \subseteq \mathcal{Z}(p)$, so [CuFi4, Proposition 3.1] implies $p(Z, \bar{Z})=0$ in $\mathcal{C}_{M(n)}$. Since $M(n)$ has a representing measure, $M(n)$ is positive and recursively generated [CuFi4, Remark 3.15], so Proposition 3.9 (iii) implies $M_{p}(n)=0$. Conversely, if $M_{p}(n)=0$, then by Proposition [3.9(1), $p(Z, \bar{Z})=0$, so [CuFi4. Proposition 3.1] implies that $\operatorname{supp} \mu \subseteq \mathcal{Z}(p)$, i.e., $\left.p\right|_{K} \equiv 0$.

In the next section we will study the relationship between successive flat extensions $M(n+1), M(n+2), \ldots$ of $M(n) \geq 0$ and the corresponding successive 
extensions of $M_{p}(n)$. As motivation for these results, we next present several examples concerning the behavior of $M_{p}(\cdot)$ with respect to positive extensions of moment matrices.

Our first example illustrates a case in which $M(1) \geq 0, M_{z}(1) \geq 0$, but for every positive extension $M(2), M_{z}(2)$ is not positive.

Example 3.12. Let $M(1)=\left(\begin{array}{lll}1 & 0 & 0 \\ 0 & 1 & 0 \\ 0 & 0 & 1\end{array}\right)$, so that $M_{z}(1)=(0) \geq 0$. For any positive extension $M(2)$, we have

$$
M_{z}(2)=\left(\begin{array}{lll}
\gamma_{01} & \gamma_{02} & \gamma_{11} \\
\gamma_{11} & \gamma_{12} & \gamma_{21} \\
\gamma_{02} & \gamma_{03} & \gamma_{12}
\end{array}\right),
$$

so self-adjointness of $M_{z}(2)$ would entail $0=\gamma_{02}=\bar{\gamma}_{11}=1$. The preceding argument implies that $\gamma^{(2)}$ has no finitely atomic representing measure supported in $\mathbb{R}^{+}$; in fact, since $Z \neq \bar{Z}$ in $\mathcal{C}_{M(1)}, \gamma^{(2)}$ has no representing measure supported in $\mathbb{R}$ [CuFi4 Proposition 3.1].

We next study a complete parameterization of the flat extensions corresponding to a particular quadratic moment problem. Depending on the choice of the flat extension $M(2)$, the corresponding representing measure will be supported in the interior of the unit disk, supported in the disk with exactly one atom on the circle, or not supported in the disk at all.

Example 3.13. Let $M(1)=\left(\begin{array}{ccc}2 & i & -i \\ -i & 1 & 0 \\ i & 0 & 1\end{array}\right) \geq 0$, so that $\bar{Z}=\alpha 1+\beta Z$ with $\alpha=-i$ and $\beta=1$. CuFi5, Section 2] thus implies that the flat extensions $M(2)$ are completely parameterized by a choice of $\gamma_{12}$ of the form

$$
\gamma_{12}=\frac{1}{2} \bar{\alpha} \gamma_{11}+\frac{i t}{2 \alpha}=\frac{i}{2}-\frac{t}{2} \quad(t \in \mathbb{R}) .
$$

Corresponding to such a flat extension $M(2)[t]$ there is a 2 -atomic representing measure $\mu[t]$, which is thus a representing measure for $\gamma^{(2)}$. Theorem 2.1 now implies that in $\mathcal{C}_{M(2)[t]}$ there is a relation of the form

$$
Z^{2}=a 1+b Z
$$

Since $\{1, Z\}$ is a basis for $\mathcal{C}_{M(1)},(3.5)$ is equivalent to

$$
\left(\begin{array}{cc}
2 & i \\
-i & 1
\end{array}\right)\left(\begin{array}{l}
a \\
b
\end{array}\right)=\left(\begin{array}{l}
\gamma_{02} \\
\gamma_{12}
\end{array}\right)=\left(\begin{array}{c}
0 \\
\frac{1}{2}-\frac{t}{2}
\end{array}\right)
$$

whence $a=\frac{1}{2}+\frac{i t}{2}, b=i-t$. Now (3.5) implies

$$
\gamma_{22}=a \gamma_{20}+b \gamma_{21}=\frac{\left(t^{2}+1\right)}{2}
$$

and

$$
\gamma_{13}=a \gamma_{11}+b \gamma_{12}=\frac{\left(t^{2}-i t\right)}{2} .
$$


Let $p(z):=1-z \bar{z} ;$ then Theorem 3.5 implies

$$
\begin{aligned}
M \equiv M_{p}(2) & =\left(\begin{array}{lll}
\gamma_{00}-\gamma_{11} & \gamma_{01}-\gamma_{12} & \gamma_{10}-\gamma_{21} \\
\gamma_{10}-\gamma_{21} & \gamma_{11}-\gamma_{22} & \gamma_{20}-\gamma_{31} \\
\gamma_{01}-\gamma_{12} & \gamma_{02}-\gamma_{13} & \gamma_{11}-\gamma_{22}
\end{array}\right) \\
& =\left(\begin{array}{ccc}
1 & \frac{i+t}{2} & \frac{-i+t}{2} \\
\frac{-i+t}{2} & \frac{1-t^{2}}{2} & \frac{-t^{2}-i t}{2} \\
\frac{i+t}{2} & \frac{-t^{2}+i t}{2} & \frac{1-t^{2}}{2}
\end{array}\right) \equiv\left(\begin{array}{lll}
A & B & C
\end{array}\right) .
\end{aligned}
$$

Observe that $C=-i A+B$, so if $[M]_{2} \geq 0$, then $M$, as a flat extension of $[M]_{2}$, is also positive. Since $\operatorname{det}[M]_{2}=\frac{1}{4}-\frac{3}{4} t^{2}$, it follows that $M_{p}(2) \geq 0$ if and only if $t^{2} \leq \frac{1}{3}$. Thus, if $t^{2}>\frac{1}{3}$, then $\operatorname{supp} \mu_{t} \nsubseteq \overline{\mathbb{D}}$.

Now suppose $t^{2} \leq \frac{1}{3}$ and recall from Theorem 2.1 that supp $\mu[t]=\mathcal{Z}\left(g_{t}\right)$, where $g_{t}(z) \equiv z^{2}-b z-a=z^{2}-(i-t) z-\left(\frac{i}{2}+\frac{i t}{2}\right)$. The roots of $g_{t}$ are

$$
z_{1}=\frac{i-t+\sqrt{1+t^{2}}}{2} \quad \text { and } \quad z_{2}=\frac{i-t-\sqrt{1+t^{2}}}{2}
$$

and since $t^{2} \leq \frac{1}{3}$, it follows that $\left|z_{i}\right| \leq 1(i=1,2)$. Note also that $t^{2}<\frac{1}{3} \Leftrightarrow$ $\operatorname{rank} M_{p}(2)=2 \Leftrightarrow\left|z_{i}\right|<1(i=1,2)$ and that $t^{2}=\frac{1}{3} \Leftrightarrow \operatorname{rank} M_{p}(2)=1 \Leftrightarrow\left|z_{1}\right|<1$ and $\left|z_{2}\right|=1$ if $t=\frac{1}{\sqrt{3}}$, and $\left|z_{1}\right|=1$ and $\left|z_{2}\right|<1$ if $t=-\frac{1}{\sqrt{3}}$.

These results are in keeping with our main results. Indeed, Theorem $1.1 \mathrm{im}$ plies that since $M(1) \geq 0$ admits a flat extension $M(2)$ for which $M_{p}(2) \geq 0$, then there exists a representing measure supported in the disk with exactly $\operatorname{rank} M(1)-\operatorname{rank} M_{p}(2)\left(=2-\operatorname{rank} M_{p}(2)\right)$ atoms in the circle.

The following example illustrates cases in which $M_{p}(n)=0$ does not imply $\operatorname{supp} \mu \subseteq \mathcal{Z}(p)$ for $n<\operatorname{deg} p \leq 2 n$.

Example 3.14. Let $M(1)=\left(\begin{array}{ccc}1 & a & a \\ a & 1 & 2 a^{2}-1 \\ a & 2 a^{2}-1 & 1\end{array}\right)$, with $|a|<1$. Then $M(1) \geq 0$ and $\bar{Z}=\alpha 1+\beta Z$ with $\alpha=2 a, \beta=-1$. It thus follows from [CuFi5, Section 2] that the flat extensions $M(2)$ are determined by

$$
\gamma_{12}=\frac{1}{2} \bar{\alpha} \gamma_{11}+\frac{i t}{2 \alpha}=a+\frac{i t}{4 a} \quad(t \in \mathbb{R})
$$

Let $p(z, \bar{z}):=1-z \bar{z}$, so that $M_{p}(1)=0$. Now $M_{p}(2)=0$ entails $\gamma_{01}-\gamma_{12}=0$, i.e., $t=0$. Thus if $t \neq 0$, the measure $\mu[t]$ corresponding to the flat extension $M(2)[t]$ satisfies $M_{p}(2)[t] \neq 0$, whence supp $\mu[t] \nsubseteq \mathcal{Z}(p)$ (by Proposition 3.10(i) applied to $M(2)[t]$ ). (It is not difficult to check that if $t=0$, then $\operatorname{supp} \mu[t] \subseteq \mathcal{Z}(p)$ and $M_{p}(2)=0$, in keeping with Theorem 1.1.)

\section{EXISTENCE OF MINIMAL REPRESENTING MEASURES}

This section is devoted to the proof of Theorem 1.1 which for convenience we restate as Theorem 4.1 below. In the sequel we assume that $M(n+1)$ is a flat extension of $M(n) \geq 0$; thus Theorem 2.1 implies that there exist unique successive flat extensions $M(n+2), M(n+3), \ldots, M(\infty)$; moreover, $M(\infty)$ admits the unique representing measure $\nu[M(n+1)]$, which is a rank $M(n)$-atomic representing measure for $\gamma^{(2 n)}(c f$. (1.4) $)$. 
Let $p \equiv p(z, \bar{z}) \in \mathbb{C}[z, \bar{z}]$ and let $K_{p}:=\{z \in \mathbb{C}: p(z, \bar{z}) \geq 0\}$. The following result characterizes the existence of a minimal representing measure supported in $K_{p}$.

Theorem 4.1. Let $M(n) \geq 0$ and suppose $\operatorname{deg} p=2 k$ or $2 k-1$. There exists $a$ rank $M(n)$-atomic representing measure for $\gamma^{(2 n)}$ supported in $K_{p}$ if and only if there is some flat extension $M(n+1)$ for which $M_{p}(n+k) \geq 0$. In this case, the measure $\nu[M(n+1)]$ is a $\operatorname{rank} M(n)$-atomic representing measure supported in $K_{p}$, with precisely $\operatorname{rank} M(n)-\operatorname{rank} M_{p}(n+k)$ atoms in $\mathcal{Z}(p)$.

Note that there is no restriction on the size of $k$ in Theorem 4.1 We pause to illustrate Theorem4.1 in a case in which $k>n$, so that $M_{p}(n)$ is not even defined.

Example 4.2. Let $n=1, M(1)=\left(\begin{array}{lll}1 & 0 & 0 \\ 0 & 1 & 0 \\ 0 & 0 & 1\end{array}\right)$ and let $p(z, \bar{z})=z^{3}-1$, so that $2=k>n$. Consider the flat extension

$$
M(2)=\left(\begin{array}{llllll}
1 & 0 & 0 & 0 & 1 & 0 \\
0 & 1 & 0 & 0 & 0 & 1 \\
0 & 0 & 1 & 1 & 0 & 0 \\
0 & 0 & 1 & 1 & 0 & 0 \\
1 & 0 & 0 & 0 & 1 & 0 \\
0 & 1 & 0 & 0 & 0 & 1
\end{array}\right) .
$$

Since $Z^{2}=\bar{Z}, \bar{Z} Z=1, \bar{Z}^{2}=Z$ in $\mathcal{C}_{M(2)}, M(3)$ is uniquely determined by the column relations $Z^{3}=\bar{Z} Z=1$ and $Z^{2} \bar{Z}=Z$. Thus, using Theorem 3.5.

$$
M_{z^{3}}(3)=\overline{\bar{Z}} \overline{\bar{Z} Z}\left(\begin{array}{ccc}
Z^{2} & Z^{3} & Z^{2} \bar{Z} \\
\bar{Z}^{2} & 0 & 0 \\
0 & 1 & 0 \\
0 & 0 & 1
\end{array}\right) \text { and } M_{1}(3)=\bar{Z}\left(\begin{array}{ccc}
1 & Z & \bar{Z} \\
\bar{Z} & 0 & 0 \\
0 & 1 & 0 \\
0 & 0 & 1
\end{array}\right)
$$

whence $M_{p}(3)=(0) \geq 0$. Thus, corresponding to the flat extension $M(2)$, Theorem 4.1 guarantees the existence of a 3-atomic representing measure $\mu$ for $\gamma^{(2)}$ with $3\left(=\operatorname{rank} M(1)-\operatorname{rank} M_{p}(3)\right)$ atoms in $\mathcal{Z}(p)$. Indeed, since $Z^{3}=1$ in $C_{M(3)}$, if $z_{0}, z_{1}, z_{2}$ denote the distinct cube roots of unity, then $\mu=\frac{1}{3}\left(\delta_{z_{0}}+\delta_{z_{1}}+\delta_{z_{2}}\right)$ is the unique such representing measure ( $c f$. [CuFi4, Example 3.2]).

To prove Theorem 4.1 we require some auxiliary results concerning flat extensions. We first show how the flat extension $M(n+1)$ determines a sequence of transition matrices $W_{n, d}(d \geq 1)$, such that the unique flat extension $M(n+d+1)$ is determined from $M(n+d)$ via $W_{n, d}$. In Theorem 4.3, which is a restatement of Theorem 1.5] we then describe how the successive extensions $M_{p}(n+k+d+1)$ $(d \geq 0)$ can be computed using the same sequence of transition matrices.

Since $M(n+1)$ is a flat extension of $M(n)$, for $0 \leq j \leq n+1$, there exist scalars $w_{\bar{Z}^{0} Z^{0}, \bar{Z}^{n+1-j} Z^{j}}, \ldots, w_{\bar{Z}^{0} Z^{n}, \bar{Z}^{n+1-j} Z^{j}}, \ldots, w_{\bar{Z}^{n}} Z^{0}, \bar{Z}^{n+1-j} Z^{j}$ such that in $\mathcal{C}_{M(n+1)}$,

$$
\begin{aligned}
& \bar{Z}^{n+1-j} Z^{j}=w_{\bar{Z}^{0} Z^{0}, \bar{Z}^{n+1-j} Z^{j}} 1+w_{\bar{Z}^{0} Z, \bar{Z}^{n+1-j} Z^{j}} Z+w_{\bar{Z} Z^{0}, \bar{Z}^{n+1-j} Z^{j}} \bar{Z} \\
& +\cdots+w_{\bar{Z}^{0} Z^{n}, \bar{Z}^{n+1-j} Z^{j}} Z^{n}+\cdots+w_{\bar{Z}^{n} Z^{0}, \bar{Z}^{n+1-j} Z^{j}} \bar{Z}^{n},
\end{aligned}
$$

and the same relation automatically holds in $\mathcal{C}_{(M(n) B(n+1))}$.

Now Theorem 2.1 implies that for $d>0$, there is a unique flat extension $M(n+d+1)$ of $M(n+d)$, defined by the following relations in $\mathcal{C}_{M(n+d+1)}$ which 
derive from (4.1):

$$
\begin{aligned}
& \bar{Z}^{n+1-j} Z^{d+j}=w_{\bar{Z}^{0} Z^{0}, \bar{Z}^{n+1-j} Z^{j}} Z^{d}+w_{\bar{Z}^{0} Z, \bar{Z}^{n+1-j} Z^{j}} Z^{d+1} \\
& +w_{\bar{Z} Z^{0}, \bar{Z}^{n+1-j} Z^{j}} \bar{Z} Z^{d}+\cdots+w_{\bar{Z}^{0} Z^{n}, \bar{Z}^{n+1-j} Z^{j}} Z^{n+d} \\
& +\cdots+w_{\bar{Z}^{n} Z^{0}, \bar{Z}^{n+1-j} Z^{j}} \bar{Z}^{n} Z^{d} \quad(0 \leq j \leq n+1) .
\end{aligned}
$$

Further, in $\mathcal{C}_{M(n+1)}$, the relation

$$
\begin{aligned}
\bar{Z}^{n+1}=w_{\bar{Z}^{0} Z^{0}, \bar{Z}^{n+1}} 1+w_{\bar{Z}^{0} Z, \bar{Z}^{n+1}} Z+w_{\bar{Z} Z^{0}, \bar{Z}^{n+1}} \bar{Z} & \\
& +\cdots+w_{\bar{Z}^{0} Z^{n}, \bar{Z}^{n+1}} Z^{n}+\cdots+w_{\bar{Z}^{n} Z^{0}, \bar{Z}^{n+1}} \bar{Z}^{n}
\end{aligned}
$$

implies that in $\mathcal{C}_{M(n+d+1)}$, for $1 \leq j \leq d$,

$$
\begin{aligned}
& \bar{Z}^{n+1+j} Z^{d-j}=w_{\bar{Z}^{0} Z^{0}, \bar{Z}^{n+1}} Z^{d-j} \bar{Z}^{j}+w_{\bar{Z}^{0} Z, \bar{Z}^{n+1}} Z^{d-j+1} \bar{Z}^{j} \\
&++w_{\bar{Z} Z^{0}, \bar{Z}^{n+1}} Z^{d-j} \bar{Z}^{j+1}+\cdots+w_{\bar{Z}^{0} Z^{n}, \bar{Z}^{n+1}} Z^{n+d-j} \bar{Z}^{j} \\
&+\cdots+w_{\bar{Z}^{n} Z^{0}, \bar{Z}^{n+1}} Z^{d-j} \bar{Z}^{n+j} .
\end{aligned}
$$

Now let $W_{n, d}$ be the $\frac{(n+d+1)(n+d+2)}{2} \times(n+d+2)$ matrix in Figure 1

(Figure 11illustrates various parallel diagonals, each delineated by a pair of large black dots; each such diagonal is constant, with value given by the matrix element above and to the left of the diagonal.)

For $d \geq 0$, write

then (4.1)-(4.4) imply that

$$
M(n+d+1)=\left(\begin{array}{cc}
M(n+d) & B(n+d+1) \\
B(n+d+1)^{*} & C(n+d+1)
\end{array}\right)
$$

$$
B(n+d+1)=M(n+d) W_{n, d}
$$

whence

$$
M(n+d+1)=\left(\begin{array}{cc}
M(n+d) & M(n+d) W_{n, d} \\
W_{n, d}^{*} M(n+d) & W_{n, d}^{*} M(n+d) W_{n, d}
\end{array}\right) .
$$

We are now prepared to prove Theorem 1.5 which we restate for convenience.

Theorem 4.3. Suppose $M(n+1)$ is a flat extension of $M(n) \geq 0$ and suppose $\operatorname{deg} p=2 k$ or $2 k-1$. If $M_{p}(n+k) \geq 0$, then for each $d \geq 0$,

$$
M_{p}(n+k+d+1)=\left(\begin{array}{cc}
M_{p}(n+k+d) & M_{p}(n+k+d) W_{n, d} \\
W_{n, d}^{*} M_{p}(n+k+d) & W_{n, d}^{*} M_{p}(n+k+d) W_{n, d}
\end{array}\right) .
$$

In particular, $M_{p}(n+k+d+1)$ is a flat (positive) extension of $M_{p}(n+k+d)$.

Proof. We may assume by induction on $d \geq 0$ that $M_{p}(n+k+d) \geq 0$. Write $p=\alpha_{1} p_{1}+\cdots+\alpha_{t} p_{t}$, where $\alpha_{i} \in \mathbb{C}$ and

$$
p_{i}=\bar{z}^{r_{i}} z^{s_{i}} \cdot \bar{z}^{l_{i}} z^{m_{i}} \quad \text { with } \quad 0 \leq r_{i}+s_{i}, l_{i}+m_{i} \leq k .
$$

Since $\operatorname{deg} p=2 k$ or $2 k-1, M_{p}(n+k+d+1)$ has size $\rho \equiv \frac{(n+d+2)(n+d+3)}{2}$; thus, by (3.2),

where $M_{i} \equiv\left[M_{p_{i}}(n+k+d+1)\right]_{\rho}$.

$$
M_{p}(n+k+d+1)=\sum_{i=1}^{t} \alpha_{i} M_{i},
$$

From (4.5)-4.6) we have

$$
M(n+k+d+1)=\left(\begin{array}{cc}
M(n+k+d) & B(n+k+d+1) \\
B(n+k+d+1)^{*} & C(n+k+d+1)
\end{array}\right)
$$




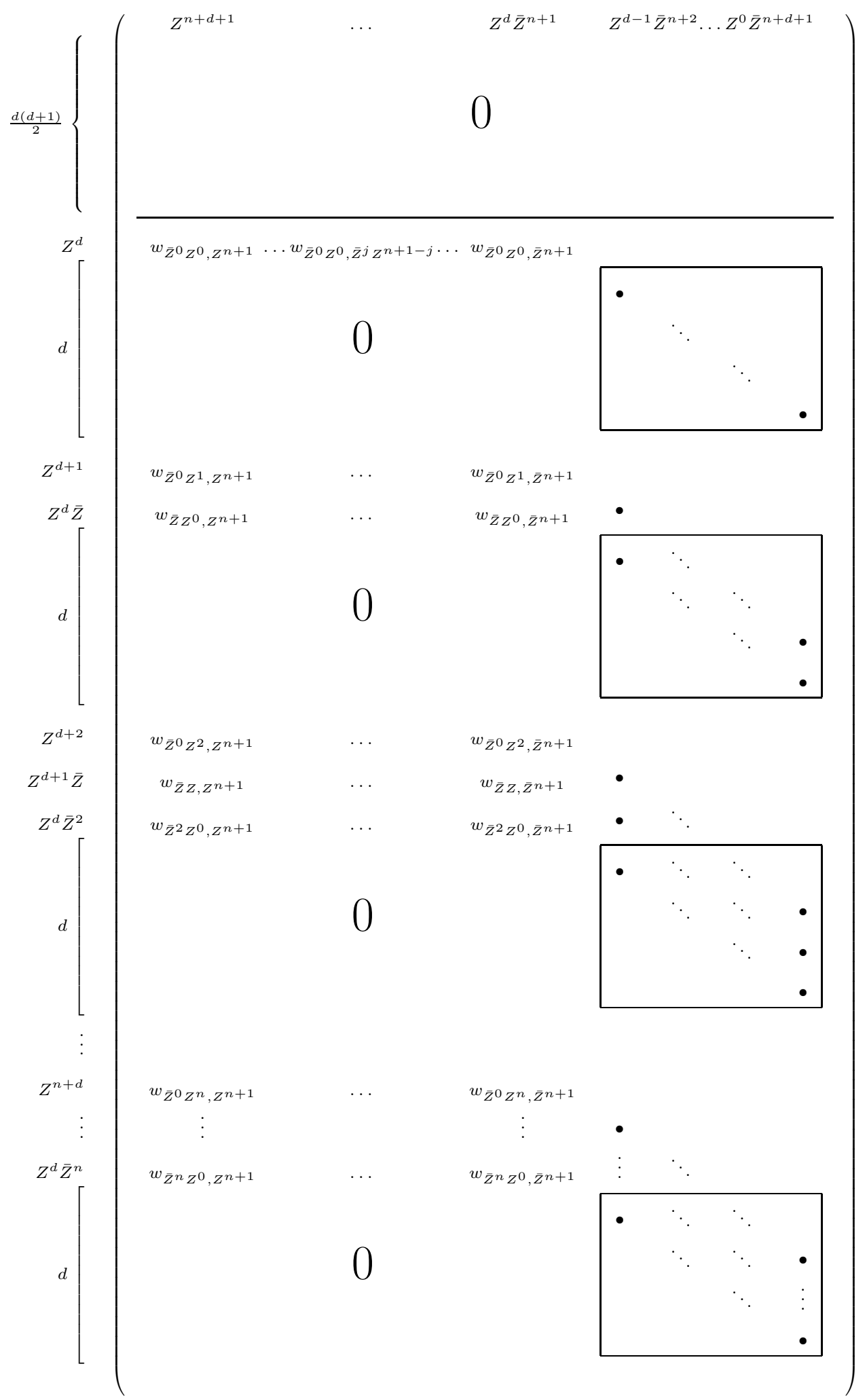

FiguRE $1 . W \equiv W_{n, d}$ 
with

$$
B(n+k+d+1)=M(n+k+d) W_{n+k, d}
$$

and

$$
C(n+k+d+1)=B(n+k+d+1)^{*} W_{n+k, d}
$$

Our next goal is to obtain a matricial description of $M_{i}$. Recall that $M_{p}(n+k+d)$ has size $\sigma \times \sigma$, where $\sigma=\frac{(n+d+1)(n+d+2)}{2}$, and that $M_{p}(n+k+d+1)$ has size $\rho \times \rho$, where $\rho=\frac{(n+d+2)(n+d+3)}{2}$. Now Theorem 3.5 implies that $M_{i}$ is the compression of $M(n+k+d+1)$ to columns indexed by the first $\rho$ multiples of $\bar{Z}^{r_{i}} Z^{s_{i}}$ and to rows indexed by the first $\rho$ multiples of $\bar{Z}^{m_{i}} Z^{l_{i}}$. In particular, it follows from Theorem 3.5 that $\left[M_{i}\right]_{\sigma}=\left[M_{p_{i}}(n+k+d)\right]_{\sigma}$, the compression of $M(n+k+d)$ to columns indexed by the first $\sigma$ multiples of $\bar{Z}^{r_{i}} Z^{s_{i}}$ and rows indexed by the first $\sigma$ multiples of $\bar{Z}^{m_{i}} Z^{l_{i}}$. We may thus denote the $\rho \times \rho$ matrix $M_{i}$ as

$$
M_{i}=\left(\begin{array}{ll}
{\left[M_{p_{i}}(n+k+d)\right]_{\sigma}} & B_{p_{i}}(n+k+d+1) \\
B_{p_{i}}^{\prime}(n+k+d+1) & C_{p_{i}}(n+k+d+1)
\end{array}\right),
$$

whence

$$
M_{p}(n+k+d+1)=\left(\begin{array}{cc}
M_{p}(n+k+d) & B_{p}(n+k+d+1) \\
B_{p}^{\prime}(n+k+d+1) & C_{p}(n+k+d+1)
\end{array}\right)
$$

where

$$
\begin{aligned}
M_{p}(n+k+d) & =\sum \alpha_{i}\left[M_{p_{i}}(n+k+d)\right]_{\sigma}, \\
B_{p}(n+k+d+1) & =\sum \alpha_{i} B_{p_{i}}(n+k+d+1), \\
B_{p}^{\prime}(n+k+d+1) & =\sum \alpha_{i} B_{p_{i}}^{\prime}(n+k+d+1), \\
C_{p}(n+k+d+1) & =\sum \alpha_{i} C_{p_{i}}(n+k+d+1) .
\end{aligned}
$$

From Theorem 3.5

$$
\left[M_{p_{i}}(n+k+d)\right]_{\sigma}={ }_{\left[\bar{Z}^{m_{i}} Z^{l_{i} ; 0, \sigma}\right]} M(n+k+d)_{\left[\bar{Z}^{r_{i}} Z^{s_{i} ; 0, \sigma}\right]}
$$

in particular, the columns of this matrix are indexed by

$$
\bar{Z}^{r_{i}+f} Z^{s_{i}+g} \quad(0 \leq f+g \leq n+d) .
$$

Thus, from Theorem 3.5, $B_{p_{i}}(n+k+d+1)$ is the compression of $M(n+k+d+1)$ to columns indexed by the next $\rho-\sigma(=n+d+2)$ multiples of $\bar{Z}^{r_{i}} Z^{s_{i}}$ and to rows indexed by the first $\sigma$ multiples of $\bar{Z}^{m_{i}} Z^{l_{i}}$. The columns of $B_{p_{i}}(n+k+d+1)$ are thus indexed by

$$
\bar{Z}^{r_{i}+f} Z^{s_{i}+g} \quad(f+g=n+d+1)
$$

so that

$$
\begin{aligned}
& B_{p_{i}}(n+k+d+1) \\
& \left.=\left[\bar{Z}^{m_{i}} Z^{l_{i} ; 0, \sigma}\right]\right]^{M(n+k+d+1)}\left[\bar{Z}^{r_{i}} Z^{s_{i} ; n+d+1+r_{i}+s_{i}, \rho-\sigma}\right] \cdot
\end{aligned}
$$

Similarly, we also have

$$
\begin{aligned}
& B_{p_{i}}^{\prime}(n+k+d+1) \\
& \quad= {\left[\bar{Z}^{m_{i}} Z^{l_{i}} ; n+d+1+m_{i}+l_{i}, \rho-\sigma\right] } \\
& \quad M(n+k+d+1)\left[\bar{Z}^{r_{i}} Z^{s_{i}} ; 0, \sigma\right]
\end{aligned}
$$


and

$$
\begin{aligned}
& C_{p_{i}}(n+k+d+1) \\
& =\left[\bar{Z}^{m_{i}} Z^{l_{i} ; n+d+1+m_{i}+l_{i}, \rho-\sigma}\right]^{M(n+k+d+1)}\left[\overline{\bar{Z}}^{r_{i} Z^{s_{i}} ; n+d+1+r_{i}+s_{i}, \rho-\sigma}\right] .
\end{aligned}
$$

We assert that

$$
B_{p_{i}}(n+k+d+1)=\left[M_{p_{i}}(n+k+d)\right]_{\sigma} W_{n, d} .
$$

To prove (4.20), let $r:=r_{i}, s:=s_{i}, l:=l_{i}, m:=m_{i}$, so that $r+s, l+m \leq k$. The columns of $\left[M_{\bar{z}^{r} z^{s} \cdot \bar{z}^{l} z^{m}}(n+k+d)\right]_{\sigma}$ are indexed by

$$
\bar{Z}^{r} Z^{s}, \bar{Z}^{r} Z^{s+1}, \bar{Z}^{r+1} Z^{s}, \ldots, \bar{Z}^{r} Z^{s+n+d}, \ldots, \bar{Z}^{r+n+d} Z^{s},
$$

and the columns of $B_{\bar{z}^{r} z^{s} \cdot \bar{z}^{l} z^{m}}(n+k+d+1)$ are indexed by

$$
\bar{Z}^{r} Z^{s+n+d+1}, \ldots, \bar{Z}^{r+n+d+1} Z^{s} \text {. }
$$

Claim. For $0 \leq j \leq n+d+1$,

$$
\bar{Z}^{r+j} Z^{s+n+d+1-j} \quad \text { in } \quad \mathcal{C}_{B_{\bar{z} r_{z} s \cdot \bar{z} l_{z} m}(n+k+d+1)}
$$

coincides with

$$
\bar{Z}^{j} Z^{n+d+1-j} \quad \text { in } \quad \mathcal{C}_{\left[M_{p_{i}}(n+k+d)\right]_{\sigma} W_{n, d}} .
$$

We may denote the columns of $\left[M_{p_{i}}(n+k+d)\right]_{\sigma} W_{n, d}$ in the same way as we index the columns of $W_{n, d}$, i.e.,

$$
\bar{Z}^{j} Z^{n+d+1-j} \quad(0 \leq j \leq n+d+1) .
$$

Case $1.0 \leq j \leq n+1$. Direct calculation shows that in $\mathcal{C}_{\left[M_{\bar{z}^{r} z^{s} \cdot \bar{z}^{l} l_{z} m}(n+k+d)\right]_{\sigma} W_{n, d}}$,

$$
\begin{aligned}
& \bar{Z}^{j} Z^{n+d+1-j}=w_{\bar{Z}^{0} Z^{0}, \bar{Z}^{j} Z^{n+1-j}} \bar{Z}^{r} Z^{s+d}+w_{\bar{Z}^{0} Z^{1}, \bar{Z}^{j} Z^{n+1-j}} \bar{Z}^{r} Z^{s+d+1} \\
& \quad+w_{\bar{Z} Z^{0}, \bar{Z}^{j} Z^{n+1-j} \bar{Z}^{r+1} Z^{s+d}+w_{\bar{Z}^{0}} Z^{2}, \bar{Z}^{j} Z^{n+1-j} \bar{Z}^{r} Z^{s+d+2}} \bar{Z}^{r+1} Z^{s+d+1}+w_{\bar{Z}^{2} Z^{0}, \bar{Z}^{j} Z^{n+1-j}} \bar{Z}^{r+2} Z^{s+d} \\
& \quad+w_{\bar{Z} Z, \bar{Z}^{j} Z^{n+1-j}} \\
& +\cdots+w_{\bar{Z}^{0} Z^{n}, \bar{Z}^{j} Z^{n+1-j}} \bar{Z}^{r} Z^{s+d+n}+\cdots+w_{\bar{Z}^{n} Z^{0}, \bar{Z}^{j} Z^{n+1-j}} \bar{Z}^{r+n} Z^{s+d} .
\end{aligned}
$$

Let $J:=n+1-j$. If we express (4.1) as

$$
\bar{Z}^{j} Z^{n+1-j}=\bar{Z}^{n+1-J} Z^{J} \equiv q_{J}(Z, \bar{Z}) \quad \text { in } \quad \mathcal{C}_{M(n+1)},
$$

then Theorem 2.1 implies that in $\mathcal{C}_{M(n+k+d+1)}$,

$$
\bar{Z}^{r+j} Z^{s+d+n+1-j}=\left(\bar{Z}^{r} Z^{s+d} q_{J}\right)(Z, \bar{Z}) .
$$

From (4.1),

$$
\begin{aligned}
q_{J}(Z, \bar{Z})= & w_{\bar{Z}^{0} Z^{0}, \bar{Z}^{n+1-J} Z^{J}} 1+w_{Z, \bar{Z}^{n+1-J} Z^{J}} Z+w_{\bar{Z}, \bar{Z}^{n+1-J} Z^{J}} \bar{Z} \\
& +\cdots+w_{\bar{Z}^{0} Z^{n}, \bar{Z}^{n+1-J} Z^{J}} Z^{n}+\cdots+w_{\bar{Z}^{n} Z^{0}, \bar{Z}^{n+1-J} Z^{J}} \bar{Z}^{n} \\
= & w_{\bar{Z}^{0} Z^{0}, \bar{Z}^{j} Z^{n+1-j}} 1+w_{Z, \bar{Z}^{j} Z^{n+1-j}} Z+w_{\bar{Z}, \bar{Z}^{j} Z^{n+1-j}} \bar{Z} \\
& +\cdots+w_{\bar{Z}^{0} Z^{n}, \bar{Z}^{j} Z^{n+1-j}} Z^{n}+\cdots+w_{\bar{Z}^{n} Z^{0}, \bar{Z}^{j} Z^{n+1-j}} \bar{Z}^{n},
\end{aligned}
$$


whence, in $\mathcal{C}_{M(n+k+d+1)}$,

$$
\begin{aligned}
& \bar{Z}^{r+j} Z^{s+d+n+1-j}=w_{\bar{Z}^{0} Z^{0}, \bar{Z}^{j} Z^{n+1-j}} \bar{Z}^{r} Z^{s+d} \\
& \quad+w_{Z, \bar{Z}^{j} Z^{n+1-j}} \bar{Z}^{r} Z^{s+d+1}+w_{\bar{Z}, \bar{Z}^{j} Z^{n+1-j}} \bar{Z}^{r+1} Z^{s+d} \\
& \quad+\cdots+w_{\bar{Z}^{0} Z^{n}, \bar{Z}^{j} Z^{n+1-j}} \bar{Z}^{r} Z^{s+d+n}+\cdots+w_{\bar{Z}^{n} Z^{0}, \bar{Z}^{j} Z^{n+1-j}} \bar{Z}^{r+n} Z^{s+d} .
\end{aligned}
$$

When (4.23) is compressed to rows indexed by the first $\sigma$ multiples of $\bar{Z}^{m} Z^{l}$, we obtain (4.22); thus $\bar{Z}^{r+j} Z^{s+d+n+1-j}$ in $\mathcal{C}_{B_{\bar{z}^{r} z^{s} \cdot \bar{z}^{l} z^{m}}(n+k+d+1)}$ coincides with $\bar{Z}^{j} Z^{n+d+1-j}$ in $\mathcal{C}_{\left[M_{\bar{z}^{r} z^{s} \cdot \bar{z}^{l} z^{m}}(n+k+d)\right]_{\sigma} W_{n, d}}$.

Case 2. $n+1<j \leq n+d+1, d \geq 1$. We express $\bar{Z}^{j} Z^{n+d+1-j}$ in $\mathcal{C}_{\left[M_{\bar{z}^{r} z^{s} \cdot \bar{z}^{l} z^{m}}(n+k+d)\right]_{\sigma} W_{n, d}}$ as follows. Let $u:=j-n-1,1 \leq u \leq d$. Then $\bar{Z}^{j} Z^{n+d+1-j}=\bar{Z}^{n+1+u} Z^{d-u}$, and by direct calculation, this equals

$$
\begin{aligned}
w_{\bar{Z}^{0} Z^{0},} \bar{Z}^{n+1} \bar{Z}^{r+u} Z^{s+d-u}+w_{Z, \bar{Z}^{n+1}} \bar{Z}^{r+u} Z^{s+d-u+1} & \\
& +w_{\bar{Z}, \bar{Z}^{n+1}} \bar{Z}^{r+u+1} Z^{s+d-u}+w_{Z^{2}, \bar{Z}^{n+1}} \bar{Z}^{r+u} Z^{s+d-u+2} \\
& +w_{\bar{Z} Z, \bar{Z}^{n+1}} \bar{Z}^{r+u+1} Z^{s+d-u+1}+w_{\bar{Z}^{2}, \bar{Z}^{n+1}} \bar{Z}^{r+u+2} Z^{s+d-u} \\
& +\cdots+w_{Z^{n}, \bar{Z}^{n+1}} \bar{Z}^{r+u} Z^{s+d-u+n}+\cdots+w_{\bar{Z}^{n}, \bar{Z}^{n+1}} \bar{Z}^{r+u+n} Z^{s+d-u} .
\end{aligned}
$$

Now, if we express (4.3) as $\bar{Z}^{n+1}=f(Z, \bar{Z})$, with $f(z, \bar{z}):=w_{1, \bar{Z}^{n+1}}+w_{Z, \bar{Z}^{n+1}} z+$ $w_{\bar{Z}, \bar{Z}^{n+1}} \bar{z}+\cdots+w_{Z^{n}, \bar{Z}^{n+1}} z^{n}+\cdots+w_{\bar{Z}^{n}, \bar{Z}^{n+1}} \bar{z}^{n}$, then in $\mathcal{C}_{M(n+k+d+1)}$,

$$
\begin{aligned}
\bar{Z}^{r+j} Z^{s+n+d+1-j}= & \bar{Z}^{r+u+n+1} Z^{s+d-u} \\
= & \left(\bar{z}^{r+u} z^{s+d-u} f\right)(Z, \bar{Z}) \\
= & w_{1, \bar{Z}^{n+1}} \bar{Z}^{r+u} Z^{s+d-u}+\cdots+w_{Z^{n} \bar{Z}^{n+1}} \bar{Z}^{r+u} Z^{s+d+n-u} \\
& \quad+\cdots+w_{\bar{Z}^{n}, \bar{Z}^{n+1}} \bar{Z}^{r+u+n} Z^{s+d-u}
\end{aligned}
$$

which coincides with (4.24) after compression to rows indexed by the first $\sigma$ multiples of $\bar{Z}^{m} Z^{l}$.

This completes the proof of the Claim.

Since $\bar{Z}^{r+j} Z^{s+n+d+1-j}$ is the $j$-th column of $B_{\bar{z}^{r} z^{s} \cdot \bar{z}^{l} z^{m}}(n+k+d+1)$ and $\bar{Z}^{j} Z^{n+d+1-j}$ is the $j$-th column of $\left[M_{p_{i}}(n+k+d)\right]_{\sigma} W_{n, d}, 0 \leq j \leq n+d+1$, it now follows that (4.20) holds.

We next assert that

$$
\begin{aligned}
B_{p_{i}}^{\prime}(n+k+d+1) & =B_{\bar{p}_{i}}(n+k+d+1)^{*} \\
& =W_{n, d}^{*}\left[M_{p_{i}}(n+k+d)\right]_{\sigma} .
\end{aligned}
$$

From (4.20), $B_{p_{i}}(n+k+d+1)=\left[M_{p_{i}}(n+k+d)\right]_{\sigma} W_{n, d}$, and thus

$$
\begin{aligned}
B_{\bar{p}_{i}}(n+k+d+1) & =\left[M_{\bar{p}_{i}}(n+k+d)\right]_{\sigma} W_{n, d} \\
& =\left[M_{p_{i}}(n+k+d)^{*}\right]_{\sigma} W_{n, d} \\
& =\left[M_{p_{i}}(n+k+d)\right]_{\sigma}^{*} W_{n, d},
\end{aligned}
$$

whence

$$
B_{\bar{p}_{i}}(n+k+d+1)^{*}=W_{n, d}^{*}\left[M_{p_{i}}(n+k+d)\right]_{\sigma} .
$$


To complete the proof of (4.26), it suffices to show that

$$
B_{p_{i}}^{\prime}(n+k+d+1)^{*}=B_{\bar{p}_{i}}(n+k+d+1) .
$$

From Lemma 3.3 and (4.18), and since $M(n+k+d+1) \geq 0$, we have

$$
\begin{aligned}
& B_{p_{i}}^{\prime}(n+k+d+1)^{*}=\left({ }_{\left[\bar{Z}^{m_{i}} Z^{\left.l_{i} ; n+d+1+m_{i}+l_{i}, \rho-\sigma\right]}\right.} M(n+k+d+1)_{\left[\bar{Z}^{r_{i}} Z^{\left.s_{i} ; 0, \sigma\right]}\right.}\right)^{*}
\end{aligned}
$$

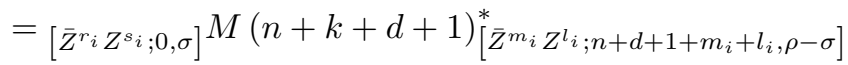

$$
\begin{aligned}
& =\left[\bar{Z}^{r_{i}} Z^{\left.s_{i} ; 0, \sigma\right]}\right]^{M(n+k+d+1)}\left[\bar{Z}^{m_{i}} Z^{l_{i} ; n+d+1+m_{i}+l_{i}, \rho-\sigma}\right] \\
& =B_{\bar{p}_{i}}(n+k+d+1)
\end{aligned}
$$

(by (4.17) applied to $\bar{p}_{i}=\bar{z}^{m_{i}} z^{l_{i}} \cdot \bar{z}^{s_{i}} z^{r_{i}}$ ).

Since the columns of $\left(\begin{array}{c}{\left[M_{p_{i}}(n+k+d)\right]_{\sigma}} \\ B_{p_{i}}^{\prime}(n+k+d+1)\end{array}\right)$ are indexed by $\bar{Z}^{r_{i}+u} Z^{s_{i}+v}(0 \leq u+v \leq$ $n+d)$ and the columns of $\left(\begin{array}{c}B_{p_{i}}(n+k+d+1) \\ C_{p_{i}}(n+k+d+1)\end{array}\right)$ are indexed by $\bar{Z}^{r_{i}+u} Z^{s_{i}+v}(u+v=$ $n+d+1)(c f$. (4.17)-(4.19), it follows from (4.11)-(4.25) (especially (4.23) and $(4.25))$ that

$$
\left(\begin{array}{c}
{\left[M_{p_{i}}(n+k+d)\right]_{\sigma}} \\
B_{p_{i}}^{\prime}(n+k+d+1)
\end{array}\right) W_{n, d}=\left(\begin{array}{c}
B_{p_{i}}(n+k+d+1) \\
C_{p_{i}}(n+k+d+1)
\end{array}\right),
$$

whence (4.12) implies

$$
\left(\begin{array}{c}
M_{p}(n+k+d) \\
B_{p}^{\prime}(n+k+d+1)
\end{array}\right) W_{n, d}=\left(\begin{array}{c}
B_{p}(n+k+d+1) \\
C_{p}(n+k+d+1)
\end{array}\right) .
$$

Now (4.15), (4.26), and (4.13) imply

$$
\begin{aligned}
B_{p}^{\prime}(n+k+d+1) & =\sum \alpha_{i} B_{p_{i}}^{\prime}(n+k+d+1) \\
& =W_{n, d}^{*} \sum \alpha_{i}\left[M_{p_{i}}(n+k+d)\right]_{\sigma} \\
& =W_{n, d}^{*} M_{p}(n+k+d),
\end{aligned}
$$

whence (4.12) and (4.28) yield

$$
M_{p}(n+k+d+1)=\left(\begin{array}{cc}
M_{p}(n+k+d) & M_{p}(n+k+d) W_{n, d} \\
W_{n, d}^{*} M_{p}(n+k+d) & W_{n, d}^{*} M_{p}(n+k+d) W_{n, d}
\end{array}\right),
$$

which is (4.7); in particular, since $M_{p}(n+k+d) \geq 0$, then $M_{p}(n+k+d+1)$ is a flat, positive extension of $M_{p}(n+k+d)$. This completes the proof of Theorem 4.3.

Proof of Theorem 4.1. Assume first that $\mu$ is a $\operatorname{rank} M(n)$-atomic representing measure, with supp $\mu \subseteq K_{p}$. By [CuFi4, Theorem 5.13], $M(n)$ admits a flat extension $M(n+1)$, which in turn gives rise to a unique flat extension $M(\infty)$. By Theorem 2.1 (or [CuFi4 Corollary 5.14]), associated to $M(\infty)$ there is a unique measure $\nu \equiv \nu(M[n+1])$, with $\operatorname{card} \operatorname{supp} \nu=\operatorname{rank} M(n+1)=\operatorname{rank} M(n)=\operatorname{card} \operatorname{supp} \mu$. Since $M(\infty)[\mu]$ is a flat extension of $M(n)$ with representing measure $\mu$, it follows that $\mu=\nu$. Thus $\mu$ is a representing measure for $M(n+k)$, and since supp $\mu \subseteq K_{p}$, (3.3) implies that $M_{p}(n+k) \geq 0$.

Conversely, suppose $M(n)$ admits a flat extension $M(n+1)$ for which $M_{p}(n+k) \geq 0$ (cf. Theorem 2.1). Repeated application of Theorem 4.3 implies 
that $M_{p}(\infty)$ is a flat (positive) extension of $M_{p}(n+k)$. Thus, for $f, g \in \mathbb{C}[z, \bar{z}]$, we have

$$
\Lambda_{\gamma}(f \bar{g})=\langle M(\infty) \hat{f}, \hat{g}\rangle
$$

and

$$
\Lambda_{\gamma}(p f \bar{g})=\left\langle M_{p}(\infty) \hat{f}, \hat{g}\right\rangle .
$$

From [CuFi4, Theorem 4.7], there exists a unique representing measure for $M(\infty)$, which is $\operatorname{rank} M(n)$-atomic, say

$$
\mu \equiv \sum_{i=1}^{r} \rho_{i} \delta_{z_{i}}
$$

where $r:=\operatorname{rank} M(n), \rho_{i}>0, z_{i} \in \mathbb{C}$.

Thus

$$
\langle M(\infty) \hat{f}, \hat{g}\rangle=\int f \bar{g} d \mu=\sum \rho_{i} f\left(z_{i}, \bar{z}_{i}\right) \bar{g}\left(z_{i}, \bar{z}_{i}\right)
$$

and

$$
\left\langle M_{p}(\infty) \hat{f}, \hat{g}\right\rangle=\int p f \bar{g} d \mu=\sum \rho_{i} p\left(z_{i}, \bar{z}_{i}\right) f\left(z_{i}, \bar{z}_{i}\right) \bar{g}\left(z_{i}, \bar{z}_{i}\right)
$$

Fix $i_{0}, 1 \leq i_{0} \leq r$, and let $f \equiv f_{i_{0}} \in \mathbb{C}[z]$ denote a polynomial such that $f_{i_{0}}\left(z_{i_{0}}\right)=1$ and $f_{i_{0}}\left(z_{j}\right)=0$ for $j \neq i_{0}$. Since $M_{p}(\infty) \geq 0$,

$$
0 \leq\left\langle M_{p}(\infty) \hat{f}, \hat{f}\right\rangle=\rho_{i_{0}} p\left(z_{i_{0}}, \bar{z}_{i_{0}}\right) .
$$

Since $\rho_{i_{0}}>0$, it follows that $p\left(z_{i_{0}}, \bar{z}_{i_{0}}\right) \geq 0$. Thus supp $\mu \subseteq K_{p} \equiv\{z: p(z, \bar{z}) \geq 0\}$.

Now $p d \mu \geq 0$, and (4.32) shows that $M_{p}(\infty)$ is the corresponding moment matrix [CuFi4, Theorem 4.7]. By [CuFi4, Chapter 4], it follows that card supp $p d \mu=$ $\operatorname{rank} M_{p}(\infty) ;$ similarly, $\operatorname{rank} M(\infty)=$ card $\operatorname{supp} \mu$. Now

$$
\operatorname{supp} p d \mu=\left\{z_{i} \in \operatorname{supp} \mu: p\left(z_{i}, \bar{z}_{i}\right)>0\right\}=\operatorname{supp} \mu \backslash \mathcal{Z}(p) .
$$

We thus have

$$
\begin{aligned}
\operatorname{rank} M(n)-\operatorname{rank} M_{p}(n+k) & =\operatorname{rank} M(\infty)-\operatorname{rank} M_{p}(\infty) \\
& =\operatorname{card} \operatorname{supp} \mu-\operatorname{card} \operatorname{supp} p d \mu \\
& =\operatorname{card} \operatorname{supp} \mu \cap \mathcal{Z}(p) ;
\end{aligned}
$$

thus $\mu$ has $\operatorname{rank} M(n)-\operatorname{rank} M_{p}(n+k)$ atoms in $\mathcal{Z}(p)$. The proof of Theorem 4.1 is now complete.

For the reader's convenience, we now restate Theorem 1.6. which is an immediate consequence of Theorem 4.1 and its proof.

Theorem 4.4. There exists a rank $M(n)$-atomic representing measure for $\gamma^{(2 n)}$ supported in $K_{\mathcal{P}}:=\left\{z \in \mathbb{C}: p_{i}(z, \bar{z}) \geq 0,1 \leq i \leq m\right\}$ if and only if $M(n) \geq 0$ and there is some flat extension $M(n+1)$ for which $M_{p_{i}}\left(n+k_{i}\right) \geq 0(1 \leq i \leq$ $m)$. In this case, the measure $\nu[M(n+1)]$ is a rank $M(n)$-atomic representing measure supported in $K_{\mathcal{P}}$, with precisely $\operatorname{rank} M(n)-\operatorname{rank} M_{p_{i}}\left(n+k_{i}\right)$ atoms in $\mathcal{Z}\left(p_{i}\right)(1 \leq i \leq m)$. 
We illustrate Theorem 4.1 with a particular quartic moment problem for the closed unit disk.

Example 4.5. Let

$$
\begin{aligned}
M & \equiv M(2) \\
& :=\left(\begin{array}{cccccc}
2 & i & -i & 0 & 1 & 0 \\
-i & 1 & 0 & a+\frac{i}{2} & a-\frac{i}{2} & a-\frac{i}{2} \\
i & 0 & 1 & a+\frac{i}{2} & a+\frac{i}{2} & a-\frac{i}{2} \\
& & & & & \\
0 & a-\frac{i}{2} & a-\frac{i}{2} & c & -i\left(a-\frac{i}{2}\right)+c & -2 i\left(a-\frac{i}{2}\right)+c \\
1 & a+\frac{i}{2} & a-\frac{i}{2} & i\left(a+\frac{i}{2}\right)+c & c & -i\left(a-\frac{i}{2}\right)+c \\
0 & a+\frac{i}{2} & a+\frac{i}{2} & 2 i\left(a+\frac{i}{2}\right)+c & i\left(a+\frac{i}{2}\right)+c & c
\end{array}\right) .
\end{aligned}
$$

We will describe the $\operatorname{rank} M(2)$-atomic representing measures supported in the closed unit disk $\overline{\mathbb{D}}$. With $\alpha=-i, \beta=1$, we have

$$
\bar{Z}=\alpha 1+\beta Z, \bar{Z} Z=\alpha Z+\beta Z^{2}, \bar{Z}^{2}=\alpha^{2} 1+2 \alpha \beta Z+\beta^{2} Z^{2} .
$$

Now $[M]_{2}>0$, and $[M]_{3}(\geq 0)$ is a flat extension of $[M]_{2}$. Let $r=-i\left(a+\frac{i}{2}\right)$, $s=2\left(a+\frac{i}{2}\right)$, so that $\left[Z^{2}\right]_{(3)}=r[1]_{(3)}+s[Z]_{3}$. Thus $[M]_{4} \geq 0$ if and only if $c \geq r \cdot 0+s\left(a-\frac{i}{2}\right)=2 a^{2}+\frac{1}{2}$. With $c \geq 2 a^{2}+\frac{1}{2},[M]_{5} \geq 0$, since $[M]_{5}$ is a flat extension of $[M]_{4}$, and $M=[M]_{6} \geq 0$, since $[M]_{6}$ is a flat extension of $[M]_{5}$ (by 4.33).

We consider first the case when $M(2) \geq 0$ and $\operatorname{rank} M(2)=2$, i.e., $c=2 a^{2}+\frac{1}{2}$. In this case, $\gamma^{(4)}$ is of flat data type, so [CuFi4, Corollary 5.14] implies that there exists a unique representing measure $\mu$, which is 2-atomic. Let $p(z, \bar{z}):=1-z \bar{z}$. Since $M(2)$ is a flat extension of $M(1)$, Theorem 4.1 implies supp $\mu \subseteq \overline{\mathbb{D}}$ if and only if $M_{p}(2) \geq 0$, and a calculation shows that this occurs precisely when $a^{2} \leq \frac{1}{12}$. If $a^{2}=\frac{1}{12}, \operatorname{rank} M_{p}(2)=1$, so there is one atom on the unit circle, while if $a^{2}<\frac{1}{12}$, rank $M_{p}(2)=2$, so both atoms are in the open unit disk.

Now let $c>2 a^{2}+\frac{1}{2}$, so that $M$ is positive, $\operatorname{rank} M=3$, and $M$ is recursively generated (by (4.33)). Since $\bar{Z}=\alpha 1+\beta Z$, it follows from [CuFi5, proof of Theorem 2.1 ] that any flat extension $M(3)$ of $M(2)$ is completely determined by a choice of $\gamma_{23}$ of the form

$$
\gamma_{23}=\frac{1}{2} \bar{\alpha} \gamma_{22}+\frac{i t}{2 \alpha}=\frac{i}{2} c-\frac{t}{2} \quad(t \in \mathbb{R}) .
$$

It follows from Theorem 2.1 that in any such flat extension $M(3)[t]$, there exists a column relation of the form

$$
Z^{3}=c_{0} 1+c_{1} Z+c_{2} Z^{2}
$$

where

$$
\left\{1, Z, Z^{2}\right\} M(2)_{\left\{1, Z, Z^{2}\right\}}\left(c_{0}, c_{1}, c_{2}\right)^{t}=\left(\gamma_{03}, \gamma_{13}, \gamma_{23}\right)^{t}
$$


We have

$$
\begin{aligned}
& c_{0}=\frac{2 a+8 a^{3}-12 a c-2 t}{4\left(1+4 a^{2}-2 c\right)}+\frac{i}{4} \frac{\left(1+4 a^{2}-6 c+8 c^{2}+4 a t\right)}{\left(1+4 a^{2}-2 c\right)}, \\
& c_{1}=\frac{i(4 a c+t)}{-1-4 a^{2}+2 c}+\frac{2\left(-c+2 c^{2}+a t\right)}{\left(-1-4 a^{2}+2 c\right)}, \\
& c_{2}=\frac{i}{2} \frac{\left(3+12 a^{2}-6 c\right)}{\left(1+4 a^{2}-2 c\right)}+\frac{(8 a c+2 t)}{2\left(1+4 a^{2}-2 c\right)},
\end{aligned}
$$

and the support of the representing measure $\mu_{t}$ corresponding to $M(3)[t]$ consists of the three distinct roots of

$$
g_{t}(z):=z^{3}-\left(c_{0}+c_{1} z+c_{2} z^{2}\right) .
$$

We seek to characterize when supp $\mu_{t}$ is contained in the unit disk. From (4.33), in $\mathcal{C}_{M(3)[t]}$ we have

$$
Z^{2} \bar{Z}=\alpha Z^{2}+\beta Z^{3} \quad \text { and } \quad Z \bar{Z}^{2}=\alpha^{2} Z+2 \alpha \beta Z^{2}+\beta^{2} Z^{3} .
$$

Since, by Theorem 3.5,

$$
\begin{aligned}
& N \equiv M_{p}(3)=\left\{1, Z, \bar{Z}, Z^{2}, Z \bar{Z}, \bar{Z}^{2}\right\} M(3)_{\left\{1, Z, \bar{Z}, Z^{2}, Z \bar{Z}, \bar{Z}^{2}\right\}} \\
&-\left\{Z, Z^{2}, Z \bar{Z}, Z^{3}, Z^{2} \bar{Z}, Z \bar{Z}^{2}\right\} \\
& M(3)_{\left\{Z, Z^{2}, Z \bar{Z}, Z^{3}, Z^{2} \bar{Z}, Z \bar{Z}^{2}\right\}},
\end{aligned}
$$

it follows from (4.33) and (4.36) that the first, second, and fourth columns of $N$ $\operatorname{span} \mathcal{C}_{N}$. Thus, to establish $N \geq 0$, it suffices to show $N_{\{1,2,4\}} \geq 0$. Now $[N]_{1}>0$, and $[N]_{2} \geq 0$ if and only if $\operatorname{det}[N]_{2} \equiv \frac{3}{4}-a^{2}-c \geq 0$.

We first consider the case $c=\frac{3}{4}-a^{2}$. In this case, $N_{\{1,2,4\}} \geq 0$ if and only if $t=\frac{-5 a-4 a^{3}}{2}$, whence $N \geq 0$ and $\operatorname{rank} N=1$. Theorem 4.1 implies that $\mu_{t}$ has 2 atoms on the unit circle and one atom in the open disk.

Next, suppose $\frac{3}{4}-a^{2}-c>0$ (i.e., $\operatorname{rank} M_{p}(3)>1$ ); thus $2 a^{2}+\frac{1}{2}<c<\frac{3}{4}-a^{2}$, which entails $a^{2}<\frac{1}{12}$. Thus, under these conditions, $N \geq 0 \Leftrightarrow \operatorname{det} N_{\{1,2,4\}} \geq 0$. A calculation shows that $\operatorname{det} N_{\{1,2,4\}}=\frac{A t^{2}+B t+C}{32\left(1+4 a^{2}-2 c\right)}$, where

$$
\begin{aligned}
A= & 4-48 a^{2} \quad\left(>0, \text { since } a^{2}<\frac{1}{12}\right), \\
B= & -64 a-256 a^{3}+256 a c-192 a c^{2}, \\
C= & -27-212 a^{2}-400 a^{4}+64 a^{6}+180 c+736 a^{2} c+64 a^{4} c \\
\quad & \quad 444 c^{2}-560 a^{2} c^{2}+480 c^{3}-192 c^{4} .
\end{aligned}
$$

Now $\delta:=B^{2}-4 A C=48\left(-1-4 a^{2}+2 c\right)^{2}\left(-3+4 a^{2}+4 c\right)^{2}>0$, and since $A>0$ and $1+4 a^{2}-2 c<0$, it follows that $N \geq 0$ if and only if $t_{1} \leq t \leq t_{2}$, where $t_{1}:=\frac{-B-\sqrt{\delta}}{2 A}$ and $t_{2}:=\frac{-B+\sqrt{\delta}}{2 A}$; moreover, $\operatorname{rank} M_{p}(3)=3$ if $t_{1}<t<t_{2}$ and $\operatorname{rank} M_{p}(3)=2$ if $t=t_{1}$ or $t=t_{2}$.

Theorem 4.1 implies that if $t_{1}<t<t_{2}$, then supp $\mu_{t}$ is contained in the open disk, while if $t=t_{1}$ or $t=t_{2}, \mu_{t}$ has two atoms in the open disk and one atom on the unit circle. We illustrate this with $a=\frac{1}{4}, c=\frac{21}{32}$, whence $t_{1}=-\frac{(85+2 \sqrt{3})}{128}$ and $t_{2}=\frac{(-85+2 \sqrt{3})}{128}$. For $t=-\frac{85}{256}$, we have $c_{0}=\frac{1}{16}-\frac{3 i}{8}, c_{1}=\frac{5}{4}-\frac{i}{8}, c_{2}=\frac{1}{8}+\frac{3 i}{2}$; $z_{0} \approx 0.8465+0.5 i, z_{1}=-0.5+0.5 i, z_{2} \approx-0.2215+0.5 i ; \rho_{0} \approx 0.501717, \rho_{1}=\frac{1}{3}$, $\rho_{2} \approx 1.16495$. For $t=t_{2}, c_{0}=-\frac{i}{16}(6+i+(1+2 i) \sqrt{3}), c_{1}=\frac{10-i+(1+2 i) \sqrt{3}}{8}, c_{2}=$ 
$\frac{(1+12 i-2 \sqrt{3})}{8} ; z_{0} \approx 0.836768+0.5 i, z_{1} \approx-0.866025+0.5 i, z_{2} \approx-0.278755+0.5 i ;$ in this case, $\left|z_{1}\right|=1,\left|z_{0}\right|,\left|z_{2}\right|<1$.

\section{Truncated $K$-MOMEnT PRoblems in $\mathbb{R}$}

In this section we adapt Theorems 4.1 and 4.4 to the context of truncated moment problems on the real line, and we thereby obtain new characterizations for solubility in the truncated moment problems of Stieltjes $(K=[0,+\infty))$, Hamburger $(K=\mathbb{R})$, and Hausdorff $(K=[a, b])$ CuFi1], $\mathrm{KrNu}$, [ShTa]. For moment problems in one real variable, the moment matrix corresponding to the real truncated moment sequence $\beta \equiv \beta^{(2 n)}: \beta_{0}, \ldots, \beta_{2 n}$, with $\beta_{0}>0$, is the Hankel matrix $H(n) \equiv\left(\beta_{i+j}\right)_{0 \leq i+j \leq 2 n}$. We recall the structure of a positive Hankel matrix $H(n)$ with $r \equiv \operatorname{rank} H(n)$ and columns $1, t, \ldots, t^{n}$.

Theorem 5.1 (cf. [CuFi1, Theorem 3.9], [Fi1, Theorem 1.2], [Fi2, Theorem 4.2]). The following are equivalent:

(i) $H(n)$ admits a positive extension $H(n+1)$;

(ii) $H(n) \geq 0$ admits a flat extension $H(n+1)$;

(iii) $H(r-1) \geq 0$, and there exist (unique) real scalars $\alpha_{0}, \ldots, \alpha_{r-1}$ such that

$$
t^{r+s}=\alpha_{0} t^{s}+\cdots+\alpha_{r-1} t^{r+s-1} \quad(0 \leq s \leq n-r) .
$$

Let $K \subseteq \mathbb{R}$; for the $K$-moment problem of $\beta^{(2 n)}$, a positive Borel measure $\mu$ supported in $K$ is a representing measure if $\beta_{j}=\int t^{j} d \mu(0 \leq j \leq 2 n)$.

In case the conditions of Theorem 5.1 hold, $H(n)$ is recursively generated in the sense of [CuFi1. Thus, if $r \leq n$, (5.1) implies that $H(r)$ admits unique successive flat (positive) Hankel extensions $H(r+s), s \geq 1$. If $r=n+1$ and $H(n) \geq 0$, then corresponding to each choice of $\beta_{2 n+1} \in \mathbb{R}$, there exists a unique flat extension $H(n+1)$, which in turn admits unique successive flat extensions $H(n+2), H(n+3), \ldots$ Thus, whether or not $H(n) \geq 0$ is singular, the flat extension $H(r)$ of $H(r-1)$ admits a unique flat (positive) extension $H(\infty)$, which in turn admits a unique ( $r$-atomic) representing measure ( $c f$. Theorem 2.1).

For $0 \leq k \leq n$ and $p \in \mathbb{C}[t]$, with $\operatorname{deg} p=2 k$ or $2 k-1$, there exists a unique matrix $H_{p}(n) \in M_{n-k+1}(\mathbb{R})$ such that

$$
\left\langle H_{p}(n) \hat{f}, \hat{g}\right\rangle=\Lambda_{\beta}(p f \bar{g}), \quad \operatorname{deg} f, g \leq n-k
$$

(where $\Lambda_{\beta}\left(\sum_{i} a_{i} t^{i}\right):=\sum_{i} a_{i} \beta_{i}, c f$. (3.2)). Thus, if $\beta$ admits a representing measure $\mu$ supported in $K_{p} \equiv\{t \in \mathbb{R}: p(t) \geq 0\}$, then $H_{p}(n) \geq 0$. We have the following analogue of Theorem 1.1.

Theorem 5.2. For $\beta \equiv \beta^{(2 n)}$, let $r:=\operatorname{rank} H(n)(\beta)$. Then $\beta$ has an $r$-atomic (minimal) representing measure supported in $K_{p}$ if and only if $H(n) \geq 0$ admits a flat extension $H(n+1)$ satisfying $H_{p}(r-1+k) \geq 0$ (relative to the unique successive flat extensions $H(r+1), H(r+2), \ldots$ of $H(r)$ in the case $k \geq 2)$.

Proof. Suppose $H(n)$ admits a flat extension, so that $H(r)$ admits unique successive flat extensions $H(r+s)(s \geq 1)$ (cf. the remarks following Theorem [5.1). Formal repetition of the proof of Theorem 1.1 applied to $H(r-1)$ instead of to $M(n)$, implies that if $H_{p}(r-1+k) \geq 0$, then there exists an $r$-atomic representing measure $\mu$ for $H(r-1)$ supported in $K_{p}$ (with precisely $r-\operatorname{rank} H_{p}(r-1+k$ ) 
atoms in $\mathcal{Z}(p))$. In the case when $r \leq n$, Theorem[5.1(iii) readily implies that $\mu$ is a representing measure for all of $\beta^{(2 n)}$.

Conversely, if $\mu$ is an $r$-atomic representing measure for $\beta$, then $H(n+1)[\mu]$ is a flat extension of $H(n)$, since $\operatorname{rank} H(n+1)[\mu] \leq \operatorname{card} \operatorname{supp} \mu=r=\operatorname{rank} H(n)=$ $\operatorname{rank} H(n)[\mu] \leq \operatorname{rank} H(n+1)[\mu]$; moreover, $H(n+1)[\mu]$ has unique flat extensions $H(n+1+j)[\mu](j \geq 1)$. Thus, if $\operatorname{supp} \mu \subseteq K_{p}$, then (5.2) implies $H_{p}(r-1+k)[\mu] \geq 0$.

Using the preceding results, we can now sharpen existence theorems for the truncated moment problems of Stieltjes, Hamburger, and Hausdorff presented in CuFi1]. In [CuFi1 Theorem 5.3] we proved that $\beta^{(2 n)}$ has a representing measure supported in $K \equiv[0,+\infty)$ if and only if $H(n) \geq 0, L(n-1) \equiv$ $\left(\gamma_{i+j+1}\right)_{0 \leq i+j+1 \leq 2 n-1} \geq 0$, and $w_{n+1} \equiv\left(\beta_{n+1}, \ldots, \beta_{2 n}\right)^{t} \in \operatorname{Ran} L(n-1)$; note that $L(n-1)=H_{x}(n)$.

Theorem 5.3. Let $r=\operatorname{rank} H(n)$. The following are equivalent for $\beta^{(2 n)}$.

(i) There exists a representing measure for $\beta$ contained in $[0,+\infty)$.

(ii) There exists an $r$-atomic (minimal) representing measure for $\beta$ contained in $[0,+\infty)$.

(iii) $H(n) \geq 0, L(n-1) \geq 0$, and $w_{n+1} \in \operatorname{Ran} L(n-1)$.

(iv) $H(n) \geq 0$ has a flat extension $H(n+1)$ satisfying $H_{x}(n+1) \geq 0$.

(v) $H(n) \geq 0$ admits a flat extension $H(n+1)$ satisfying $H_{x}(r) \geq 0$.

Proof. The equivalence (ii) $\Leftrightarrow$ (iii) $\Leftrightarrow$ (iii) is contained in CuFi1, Theorem 5.3]. The equivalence (iii) $\Leftrightarrow$ (घ) follows from Theorem 5.2 (with $p(x)=x, k=1$ ); and clearly (iv) $\Rightarrow(\mathbb{v})$. It suffices to assume $r \leq n$ and to prove $(\mathbf{v}) \Rightarrow(\mathrm{iv})$ in this case. By Theorem $[5.2$ and the remarks preceding it, $H(r)$ admits unique successive flat extensions (among them $H(n+1)$ ), and the resulting extension $H(\infty)$ has a unique representing measure, which is supported in $[0,+\infty)$; in particular, $H_{x}(n+1) \geq 0$.

The truncated Hamburger moment problem, for measures supported in $K \equiv \mathbb{R}$, corresponds to $p(x) \equiv 1, k=0$. Thus Theorems 5.1 and 5.2 immediately yield the following result.

Theorem 5.4 (cf. [CuFi1, Theorem 3.9]). Let $r:=\operatorname{rank} H(n)$. Then $\beta^{(2 n)}$ has an r-atomic (minimal) representing measure supported in $\mathbb{R}$ if and only if $H(n)$ admits a positive extension $H(n+1)$.

([CuFi1, Theorem 3.9] shows that the conditions of Theorem 5.4 are also equivalent to the existence of a representing measure for $\beta^{(2 n)}$ supported in $\mathbb{R}$.)

We next consider the truncated Hausdorff moment problem, i.e., $K=[a, b]$; thus $p_{1}(x)=b-x, p_{2}(x)=x-a, k=1$. In $\mathrm{KrNu}$ Krein and Nudelman proved that $\beta \equiv \beta^{(2 n)}$ has a representing measure supported in $[a, b]$ if and only if

$$
H(n) \geq 0 \quad \text { and } \quad(a+b) L(n-1) \geq a b H(n-1)+J(n-1),
$$

where $J(n-1)=\left(\gamma_{i+j}\right)_{i, j=1}^{n}$. It is straightforward to check that the latter inequality is equivalent to the condition $H_{p_{1} p_{2}}(n) \geq 0$. Alternately, Theorem 5.1, and an obvious extension of Theorem 5.2 to general semi-algebraic sets, yield the following criterion. 
Theorem 5.5. Let $r:=\operatorname{rank} H(n)$. Then $\beta^{(2 n)}$ has an $r$-atomic (minimal) representing measure supported in $[a, b]$ if and only if $H(n) \geq 0$ admits a flat extension $H(n+1)$ and $H_{p_{i}}(r) \geq 0(i=1,2)$, i.e., $b H(r-1) \geq H_{x}(r) \geq a H(r-1)$.

Concrete conditions (in terms of the $\beta_{i}$ ) for the existence of such a flat extension are given in Fi1]. In CuFi1 we proved that $\beta^{(2 n)}$ has a representing measure supported in $[a, b]$ if and only if there is an $r$-atomic representing measure supported in $[a, b]$, and that this occurs if and only if $H(n) \geq 0$ admits a flat extension for which $b H(n) \geq H_{x}(n) \geq a H(n)$; Theorem 5.5 thus refines this result in the case when $r \leq n$.

Added in Proof. For the Full Complex $K$-Moment Problem we would like to mention recent work of M. Putinar, B. Reznick, J. Stochel, F. Szafraniec, and F.-H. Vasilescu, in which new necessary and sufficient conditions for the existence of representing measures are presented ([PuVa1], [PuVa2], Rez], [StSz1], StSz2], Va1, Va2 ). For instance, a rather concrete Riesz-type condition is obtained in PuVa1, when $K$ is a compact semi-algebraic set. This work, together with the results in $\mathrm{PuVa2}$, is partially motivated by a new proof of the representation of a polynomial positive in $K$ as a sum of squares of rational functions, allowing as denominators only powers of $1+|z|^{2}$, a fact also reproved, using different techniques, in Rez].

\section{REFERENCES}

[Akh] N.I. Akhiezer, The classical moment problem and some related questions in analysis, Hafner Publishing Co., New York, 1965. MR 32:1518

[Atz] A. Atzmon, A moment problem for positive measures on the unit disc, Pacific J. Math. 59 (1975), 317-325. MR 53:5880

[CuFi1] R.E. Curto and L.A. Fialkow, Recursiveness, positivity, and truncated moment problems, Houston J. Math. 17 (1991), 603-635. MR 93a:47016

[CuFi2] R.E. Curto and L.A. Fialkow, Recursively generated weighted shifts and the subnormal completion problem, Integral Equations Operator Theory 17 (1993), 202-246. MR 94h:47050

[CuFi3] R.E. Curto and L.A. Fialkow, Recursively generated weighted shifts and the subnormal completion problem, II, Integral Equations Operator Theory 18 (1994), 369-426. MR 94m:47044

[CuFi4] R.E. Curto and L.A. Fialkow, Solution of the truncated complex moment problem for flat data, Mem. Amer. Math. Soc. 119, no. 568, (1996). MR 96g:47009

[CuFi5] R.E. Curto and L.A. Fialkow, Flat extensions of positive moment matrices: Relations in analytic or conjugate terms, Oper. Theory Adv. Appl. 104 (1998), 59-82. CMP 98:17

[CuFi6] R.E. Curto and L.A. Fialkow, Flat extensions of positive moment matrices: Recursively generated relations, Mem. Amer. Math. Soc., 136, no. 648 (1998). MR 99d:47015

[CuFi7] R.E. Curto and L.A. Fialkow, The quadratic moment problem for the unit disc and unit circle, preprint 1998.

[Fi1] L. Fialkow, Positivity, extensions and the truncated complex moment problem, Multivariable operator theory (Seattle, WA, 1993), Contemp. Math., vol. 185, Amer. Math. Soc., Providence, RI, 1995, pp. 133-150. MR 96g:44013

[Fi2] L. Fialkow, Minimal representing measures arising from rank-increasing moment matrix extensions, J. Operator Theory, to appear.

[Fi3] L. Fialkow, Multivariable quadrature and extensions of moment matrices, preprint 1996.

[Fug] B. Fuglede, The multidimensional moment problem, Exposition. Math. 1 (1983), 47-65. MR 85g:44010

[Hav] E.K. Haviland, On the momentum problem for distributions in more than one dimension, Amer. J. Math. 57 (1935), 562-568; II, Amer. J. Math. 58 (1936), 164-168. 
[KrNu] M.G. Krein and A.A. Nudel'man, The Markov moment problem and extremal problems, Transl. Math. Monographs, vol. 50, American Mathematical Society, Providence, R.I., 1977. MR 56:16284

[Put] M. Putinar, Positive polynomials on compact semi-algebraic sets, Indiana Univ. Math. J. 42 (1993), 969-984. MR 95h:47014

[PuVa1] M. Putinar and F.-H. Vasilescu, Problème des moments sur les compacts semialgébriques, C.R. Acad. Sci. Paris Sér. I Math. 323 (1996), 787-791. MR 97i:44007

[PuVa2] M. Putinar and F.-H. Vasilescu, Solving moment problems by dimensional extension, C. R. Acad. Sci. Paris Sér. Math. 328 (1999), 495-499. CMP 99:10

[Rez] B. Reznick, Uniform denominators in Hilbert's Seventeenth Problem, Math. Z. 220 (1995), 75-97. MR 96e:11056

[Rie] M. Riesz, Sur le problème des moments, Troisième Note, Arkiv för Matematik, Astronomi och Fysik 17 (1923), no. 16, 1-52.

[Sch] K. Schmüdgen, The K-moment problem for compact semi-algebraic sets, Math. Ann. 289 (1991), 203-206. MR 92b:44011

[ShTa] J.A. Shohat and J.D. Tamarkin, The problem of moments, American Mathematical Society, New York, 1943. MR 5:5c

[Smu] Ju. L. Smul'jan, An operator Hellinger integral, Mat. Sb. (N.S.) 49 (1959), 381-430 (Russian). MR 22:12396

[Sto] J. Stochel, private communication.

[StSz1] J. Stochel and F. Szafraniec, Moment functions on real algebraic sets, Ark. Mat. 30 (1992), 133-148. MR 93d:47032

[StSz2] J. Stochel and F. Szafraniec, The complex moment problem and subnormality: A polar decomposition approach, J. Funct. Anal. 159 (1998), 432-491. CMP 99:04

[Va1] F.-H. Vasilescu, Moment problems for multi-sequences of operators, J. Math. Anal. Appl. 219 (1998), 246-259. MR 98m:47018

[Va2] F.-H. Vasilescu, Operator moment problems, preprint 1998.

Department of Mathematics, The University of Iowa, Iowa City, Iowa 52242

E-mail address: curto@math.uiowa.edu

Department of Mathematics and Computer Science, State University of New York, New Paltz, New York 12561

E-mail address: fialkow@mcs.newpaltz.edu 